\title{
Side Effects of Pesticides on the Olive Fruit Fly Parasitoid Psyttalia concolor (Szépligeti): A Review
}

\author{
Lara A. Pinheiro ${ }^{1,2}$, Beatriz Dáder ${ }^{3}\left(\mathbb{D}\right.$, Andrea C. Wanumen ${ }^{3}$, José Alberto Pereira ${ }^{1}(\mathbb{C}$, \\ Sónia A. P. Santos ${ }^{4,5}$ and Pilar Medina ${ }^{3, * \mathbb{D}}$ \\ 1 Centro de Investigação de Montanha (CIMO), ESA, Instituto Politécnico de Bragança, Campus de Santa \\ Apolónia, 5300-253 Braganza, Portugal; lara.a.a.pinheiro@gmail.com (L.A.P.); jpereira@ipb.pt (J.A.P.) \\ 2 Escuela de Ingeniería Agraria y Forestal (EIAF), University of León, Avenida de Portugal, 24071 León, Spain \\ 3 Escuela Técnica Superior de Ingeniería Agronómica, Alimentaria y de Biosistemas, Universidad Politécnica \\ de Madrid, 28040 Madrid, Spain; beatriz.dader@upm.es (B.D.); ancawa@gmail.com (A.C.W.) \\ 4 CIQuiBio, Barreiro School of Technology, Polytechnic Institute of Setúbal, Rua Américo da Silva Marinho, \\ 2839-001 Lavradio, Portugal; sonia.santos@estbarreiro.ips.pt \\ 5 LEAF, Instituto Superior de Agronomia, Tapada da Ajuda, 1349-017 Lisbon, Portugal \\ * Correspondence: pilar.medina@upm.es; Tel.: +34-91-067-1064
}

Received: 8 October 2020; Accepted: 10 November 2020; Published: 11 November 2020

\begin{abstract}
Pesticide applications in olive orchards could alter the biological control of parasitoid Psyttalia concolor Szépligeti (Hymenoptera: Braconidae) on the key pest Bactrocera oleae Rossi (Diptera: Tephritidae). Psyttalia concolor adults can be contaminated by exposure to spray droplets, contact with treated surfaces or oral uptake from contaminated food sources. Pesticides impact both pest and parasitoid populations when they coexist in time and space, as they reduce pest numbers available for parasitoids and might cause toxic effects to parasitoids from which they need to recover. Therefore, the appropriate timing and application of selective chemical treatments provides the opportunity to incorporate this parasitoid in the IPM of B. oleae. This manuscript reviews the current literature on lethal and sublethal effects of insecticides, fungicides, herbicides, and biopesticides on $P$. concolor. Insecticides were generally more toxic, particularly organophosphates and pyrethroids, while herbicides and biopesticides had less effects on mortality and reproductive parameters. Some fungicides were quite harmful. Most of the studies were conducted in laboratory conditions, focused on reproduction as the only sublethal effect, exclusively considered the effect of a single pesticide and persistence was hardly explored. Field studies, currently quite scarce, are absolutely needed to satisfactorily assess the impact of pesticides on $P$. concolor.
\end{abstract}

Keywords: biological control; Bactrocera oleae; biopesticides; insecticides; fungicides; herbicides; integrated pest management

\section{Introduction}

Substantial quantitative and qualitative crop losses are caused by the incidence of pests and pathogens [1]. These organisms can be reduced and eliminated with the application of biological, chemical, physical, or cultural control measures. Traditionally, agriculture has extensively relied on pesticides for pest control [2]. Application of pesticides offers advantages such as efficacy and low cost, minimizing work force and reducing costly inputs as labor or fuel [3]. However, undesirable effects include side effects on non-target organisms, pest resistance to active substances, secondary pest outbreaks, or residue persistence in water, soil, and food chain [4-7].

The increasing number of farmers consciously alerting for a sustainable use of pesticides contributed to the implementation of integrated pest management (IPM) [8,9], mandatory in the 
European Union (EU) under the directive 2009/128/EC [10]. In this context, an essential component of IPM is the use of macrobial (predators or parasitoids) or microbial (bacteria, viruses, fungi, or nematodes) agents against insect and mite pests [11]. However, in some cases biological control is not as robust to reduce pest levels below the economic threshold, where the pest population or the extent of crop damage exceeds the cost of controlling the pest, despite obvious advantages such as the minimal disruption to agroecosystems. Up to date, the best strategy is the optimal management of natural enemies, with actions targeted to enhance their establishment, conservation or increase, supplemented with selective chemical treatments applied at an appropriate timing to maintain key and secondary pest populations below economic thresholds [12]. In such scenario, the application of pesticides impacts not only the pest but also the natural enemies' population. The susceptibility of natural enemies to pesticides will depend on the guild, developmental stage, sex, age, type of pesticide, application method and timing, dose rate, and mode of action [7]. According to directive 2009/128/EC, it is essential to understand the risks of pesticides and adopt more effective and selective substances [10]. Because of that, the first step for developing a successful IPM is the accurate evaluation of the potential lethal and sublethal side effects of pesticides on the biological control agents [13-15]. Although sublethal concentrations do not directly kill the exposed natural enemies, they may interfere with their physiological and behavioral traits, which can significantly affect their fitness and compromise the success of biological control $[5,16]$.

\subsection{Bactrocera oleae-Psyttalia Concolor: Compatibility between Chemical and Biological Control}

The olive fruit fly Bactrocera oleae Rossi (Diptera: Tephritidae) is a key pest of olive orchards (Olea europaea L.) worldwide, the larvae of which are strictly monophagous feeding on the olive mesocarp. Females lay their eggs in olive fruits, larvae feed off the pulp and finally pupate inside the olive or exit to pupate on the ground [17]. This causes a premature fruit drop and fruit weight loss. Moreover, microorganism growth inside the fruit increases the acidity of olive oils [18]. Not all cultivars are equally susceptible to the olive fruit fly, being the severity of the damage influenced by olive characteristics such as fruit size, color, exocarp hardness and chemical composition, geographical area, and weather conditions $[18,19]$.

Control methods are based on the use of (a) insecticide treatments (bait applications (insecticides mixed with an attractant) and cover sprays); and (b) traps [20]. Organochlorides, organophosphates and carbamates were the first insecticides used against the olive fruit fly, followed by the introduction of pyrethroids, neonicotinoids, and spinosad as bait treatment, which is recognized to be an integral component of IPM as it reduces pesticide levels and environmental impact on natural enemies and pollinators [21-25]. Kaolin works as a physical barrier that prevents egg laying in the olive fruit [26]. Although insecticides have been efficient and cost-effective, this pest has developed resistance to the most commonly used active substances [20,22,27-29]. Entomopathogenic nematodes are also effective and safe control agents against larvae and pupae in laboratory experiments [30,31], although nematode efficacy in field conditions is still a challenge. In the Mediterranean area, biological control is mainly achieved by chalcidoidea ectoparasitoids Eupelmus urozonus Dalman (Eupelmidae), Pnigalio mediterraneus Ferriere and Delucchi (Eulophidae), Eurytoma martellii Domenichini (Eurytomidae) and Cyrtoptyx latipes Rondani (Pteromalidae), and the ichneumonoidea endoparasitoid Psyttalia concolor Szépligeti (Braconidae) [32,33]. All of them are native, except $P$. concolor, whose presence is linked to historical and recent releases. Since these releases were not as effective as desirable to control B. oleae in some of the regions, at present, most of the studies are focused on conservation biological control, which seeks to contribute to pest management by supporting populations of the natural enemies present in the agroecoystems [34]. Unfortunately, up to date, the control of B. oleae still relies on the use of insecticides. Psyttalia concolor is a synovigenic-females mature eggs throughout their life-, koinobiont - the host continues its development after parasitization-, larval-pupal endoparasitoid of many Tephritidae (Diptera), released or already established in Mediterranean and Californian olive groves with varying levels of success $[17,33,35,36]$. Psyttalia concolor was originally 
described by Szépligueti from B. oleae infested olives in Tunisia in 1910. It is a member of a complex of closely related species from Africa, which also includes P. humilis and P. perproximus, which have been treated as synonyms of one another, amongst others. In fact, shortly after being described in Tunisia, it was introduced to olive-growing regions of Italy, Greece and France [17,37]. It is able to attack at least sixteen tephritids on different wild and/or cultivated plants, but only two are known as typical hosts in its native range: The olive fruit fly and the medfly Ceratitis capitata Wiedemann (Diptera: Tephritidae) [38]. This parasitoid has been used in different Mediterranean areas for the biological control of $B$. oleae by inundative and propagative releases and recently released in Californian olive-groves as a part of classical biological control programmes [17]. Parasitism rates of P. concolor are low, ranging between 22.4 and 23.4\% in Spanish organic orchards in the Balearic Islands [35]. The presence of ecological infrastructures in olive groves influences the compatibility of P. concolor with pesticides. Flowering strips, banker plants and hedgerows provide food (pollen, nectar and hemipteran honeydew), alternative hosts and refuges, which are important resources for parasitoid establishment in agroecosystems [39,40]. However, these structures should also be free of pesticides because of the possible risk of contamination. Psyttalia concolor can be contaminated by contact with pesticide droplets or residues, or oral uptake from contaminated food sources. On one hand, pesticide residues persist in plant tissues long enough to contaminate pollen and nectar [41,42], essential source of energy and protein for parasitoid survival, host foraging and reproduction [43]. Psyttalia concolor may also feed on the liquid exuded from the host, particularly important for egg maturation of synovigenic females $[44,45]$, thus increasing the risk of exposure. On the other hand, parasitoids are also exposed to pesticides by direct contact with leaves during host searching, feeding, mating, and resting activities [46-48]. Pesticides induce changes in the chemical constituents of flowering or host plants, which may decrease their nutritional value or became less attractive to parasitoids [49-51], interfere with olfactory orientation during oviposition (reduced capacity to find the host or respond to host kairomones), change foraging patterns or sex pheromonal communication $[46,47,52,53]$.

\subsection{Standard Methods for Testing Side Effects of Pesticides on Natural Enemies}

Side effects of pesticides on biological control agents have been extensively studied in the last forty years. In 1974, the working group "Pesticides and Beneficial Organisms" of the International Organization for the Biological Control (IOBC) was established with the major aim at encouraging "the development of standard methods for testing the side effects of pesticides on natural enemies" [54]. IOBC test methods are based on a sequential scheme of three levels (laboratory, semi-field, and field) [55,56]. This sequence assumes that pesticides that are harmless at laboratory level will also be safe in semi-field and field conditions, and do not need to be further evaluated. However, when a chemical is categorized as harmful in laboratory conditions, its effect cannot be inferred in the next level, and the sequential scheme must be followed until (1) it displays no negative effects at semi-field level or (2) the evaluation finishes at field conditions because the chemical was also harmful at semi-field level. Laboratory methods evaluate the lethal effect (mortality) and sublethal effects (typically reproductive parameters) of pesticide residues on an inert substrate, although topical and ingestion uptake routes have been also included in this review. Semi-field tests are performed as direct application of pesticides on plants, and field studies are carried out in semi-natural field conditions [55-57]. Pesticides are ranked for (a) laboratory studies in (1) (harmless, $<30 \%$ corrected mortality with the control treatment), (2) (slightly harmful, 30-79\%), (3) (moderately harmful, 80-98\%) and (4) (harmful, $>99 \%$ ); and (b) semi-field and field studies in (1) (harmless, <25\%), (2) (slightly harmful, 25-50\%), (3) (moderately harmful, 51-75\%), and (4) (harmful, $>75 \%$ ) [58].

This work aims at reviewing the available literature on the side effects of active substances, currently registered in the EU, tested on P. concolor, categorized as insecticides, fungicides and herbicides according to their mode of action, discussing their implications on parasitoids in IPM. Moreover, we have included the available literature concerning ecotoxicology of microbial and botanical 
compounds on this parasitoid, even if their current use in olive groves can be questioned except for Bacillus thuringiensis.

\section{Side Effects of Pesticides on Psyttalia concolor}

In addition to the insecticides registered in olive groves against $B$. oleae, other pesticides can be applied against other olive pests, such as the moth Prays oleae Bernard (Lepidoptera: Praydidae) or the scale Saissetia oleae Olivier (Hemiptera: Coccidae), fungal diseases and weeds. For instance, anthracnose (Colletotrichum spp. complex) and olive leaf spot (Venturia oleaginea (Castagne) Rossman and Crous) are two important diseases causing significant yield losses and reduction of the quality of the olive oil and table olives [59,60]. Traditionally, they were controlled with copper-based fungicides [59,61], however site specific fungicides such as difenoconazole and tebuconazole, trifloxystrobin or dodine are applied nowadays $[60,62]$. Sulfur and mancozeb are contact fungicides with protective activity used to reduce the incidence of anthracnose [61]. Thus, although insecticides would presumably cause the most damage to $P$. concolor, the complex of plant protection products applied in olive trees has been included in this review, as these compounds could also have toxic effects on this parasitoid.

\subsection{Insecticides}

Organophosphates (group 1B) [63], acetylcholinesterase inhibitors, are broad spectrum insecticides with low selectivity, high acute toxicity and environmental impact [2]. Phosmet exhibited high toxicity in glass residual contact laboratory tests, but it was harmless when the substrate was treated parasitized C. capitata pupae (Table 1) [64]. Toxicity of organophosphates has been reported for other braconids, such as Aphidius gifuensis Ashmead [65] or Diachasmimorpha longicaudata Ashmead [66].

Pyrethroids (group 3A) [63] are sodium channel modulators rapidly absorbed by the insect tegument. They have a quick action, causing hyperactivity, convulsions and an immediate "knockdown" paralysis [67]. Beta-cyfluthrin, lambda-cyhalothrin, alpha-cypermethrin, zeta-cypermethrin, and deltamethrin greatly compromised $P$. concolor survival when females were exposed to residual contact and ingestion tests, but emergence was not affected when parasitized pupae were treated (Table 1) [64,68]. Short-term mortality of adults due to pyrethroids [65,66,69-72] and negative sublethal effects on larval and pupal development, fecundity, sex-ratio or oviposition [70,73-75] have been well documented in families Aphelinidae, Encyrtidae, Braconidae, Mymaridae, Trichogrammatidae, and Scelionidae. Pyrethroids can interfere with the mobility and orientation of parasitoids searching for food sources or host plants [76]. Other studies report that adults surviving to residual exposure retain their ability to orient to host odors [77], or that impaired foraging and orientation can be recovered after exposure [46,52]. As far as side effects are concerned, pyrethroids should not be recommended in IPM programs including $P$. concolor due to the high risk of mortality and interference with multiple behavioral functions [5].

Neonicotinoid imidacloprid (group 4A) [63] is a nicotinic acetylcholine receptor (nAChR) competitive modulator, which blocks the transmission of stimuli in the insect nervous system. Imidacloprid was harmless for P. concolor females in bait spraying but caused high mortality and sublethal effects on parasitization rate and progeny when applied as cover spray in glass surface or semi-field (Table 1) [78,79]. This revealed that ecological selectivity may result through the use of bait treatment. High toxicity of imidacloprid and moderate toxicity of acetamiprid have been reported with residues of up to 28 days in other braconids [65,66,72,75].

Spinosad (group 5) [63] is a combination of two fermentation factors, spinosyns A and D, produced by the actinomycete Saccharopolyspora spinosa [80]. Spinosyns disrupt nicotinic acetylcholine receptors and present high selectivity and reduced risks to the environment $[80,81]$. However, evidence about the compatibility with natural enemies is still inconclusive, particularly parasitoids, as acute lethal and multiple sublethal effects have been identified [82]. Spinosad is usually harmless for predators but moderately harmful for parasitoids [81]. Spinosad was harmful to P. concolor via ingestion, residual contact and topical application in laboratory conditions, but not in bait spraying of olive 
leaves (Table 1) [78,83]. Similarly, acute toxicity was found for braconids Bracon nigricans Szépligeti, A. gifuensis and Aphidius colemani Viereck [65,75,84], but not for D. longicaudata [66]. Bait spraying in olive orchards had no harmful effects on braconids Fopius arisanus Sonan and Psyttalia fletcheri Silvestri [84]. Spinosad residues degrade quickly, with little residual toxicity after 3-7 days post-application [66,81], although high toxicity has also been reported after 10 days [85].

Insect growth regulators (IGRs) interfere with development and reproduction [24]. Fenoxycarb (group 7B) [63] presents low soil mobility, non-accumulation and quick degradation. Potential toxicity on non-target insects has already been reviewed [86]. Fenoxycarb did not affect the longevity and emergence of P. concolor (Table 1) $[64,87]$. More recently, it was found that fenoxycarb was compatible with Eretmocerus eremicus Rose and Zolnerowich (Hymenoptera: Aphelinidae) [88], but slightly harmful to Encarsia formosa Gahan (Hymenoptera: Aphelinidae) [89]. Although it is important to increase the knowledge on P. concolor, it could be a rational candidate for IPM. Pyriproxyfen (group 7C) [63], was slightly harmful to P. concolor survival but harmless for its reproduction (Table 1) [90]. In contrast, pyriproxyfen caused high mortality on E. eremicus and E. formosa larvae and pupae [88,89]. A recent study showed a strong evidence of vertical transmission of pyriproxyfen from the treated female to the egg of parasitoid Trissolcus japonicus (Hymenoptera: Scelionidae) [91].

Diflubenzuron, an inhibitor of chitin biosynthesis type 0 (group 15) [63] drastically decreased P. concolor emergence when C. capitata larvae were fed at $0.2 \mathrm{~g}$ a.i. $/ \mathrm{kg}$ diet compared to $0.02 \mathrm{~g}$ a.i. $/ \mathrm{kg}$ diet (Table 1) [87]. A similar effect was reported in braconid Apanteles melanoscelus Ratzeburg [92].

Tebufenozide, an ecdysone receptor agonist (group 18) [63], decreased P. concolor emergence by $35 \%$ when the host larvae were treated at $6 \mathrm{~g}$ a.i. $/ \mathrm{kg}$ diet compared to $0.6 \mathrm{~g}$ a.i. $/ \mathrm{kg}$ diet (Table 1) [87]. On the contrary, it was completely harmless at a lower concentration by ingestion, residual contact or topical application (Table 1) [83]. Methoxyfenozide, another ecdysone agonist, was harmless to braconid A. gifuensis [65]. Therefore, it is important to clarify the compatibility of IGRs under field conditions.

The target protein responsible for the biological activity of azadirachtin, a limonoid tetranor-triterpenoid chemical derived from the neem tree, is unknown [63]. Azadiracthin impaired the beneficial capacity of $P$. concolor females when it was applied via treated host, residual contact or ingestion (Table 1) [83,87]. Particularly, it provoked a large reduction in the longevity, which might be related to the reported antifeeding effect of this product. Azadirachtin fresh residues also produced lethal and sublethal effects on braconids B. nigricans and D. longicaudata; however, toxicity decreased rapidly after $7-10$ days $[66,85]$.

Kaolin is a white aluminosilicate clay, non-porous, low-abrasive, and chemically inert over a wide $\mathrm{pH}$ range $[63,93]$. Kaolin was harmless to $P$. concolor at laboratory and semi-field conditions (Table 1) $[26,78,94]$. A reduction of abundance and diversity in the arthropod community of kaolin-treated olive groves has been highlighted [95-97], probably due to its deterrent effect [93]. As a general conclusion, kaolin is a recommendable pest control product in olive groves [98-100], with a low impact on non-target organisms [95,97]. 
Table 1. Toxicity of insecticides, currently registered in the European Union, tested on Psyttalia concolor adults.

\begin{tabular}{|c|c|c|c|c|c|c|c|c|c|c|c|}
\hline Active Ingredient (a.i.) & $\operatorname{MoA}^{1}$ & Trademark & Concentration & Exposure $^{2}$ & Substrate & Mortality $^{3}$ & Emergence $^{5}$ & $\begin{array}{c}\text { Attacked } \\
\text { Hosts (\%) }\end{array}$ & $\begin{array}{c}\text { Progeny } \\
(\%)\end{array}$ & $\begin{array}{l}\text { Longevity } \\
\text { (Days) }\end{array}$ & Reference \\
\hline \multirow{2}{*}{ Phosmet } & \multirow{2}{*}{$1 \mathrm{~B}$} & \multirow{2}{*}{$\operatorname{Imidan}^{\circledR}, \mathrm{ICI}$} & \multirow{2}{*}{$0.125 \%$ a.i. } & $\mathrm{R}$ & glass & $4(\mathrm{IOBC})$ & & & & & \multirow{2}{*}{ [64] } \\
\hline & & & & $\mathrm{T}$ & parasitized pupa & & 1 (IOBC) & & & & \\
\hline \multirow{2}{*}{ beta-Cyfluthrin } & \multirow{2}{*}{$3 \mathrm{~A}$} & \multirow{2}{*}{ Bulldock ${ }^{\circledR}$, Nufarm } & \multirow{2}{*}{$0.03 \mathrm{~mL} / 100 \mathrm{~mL}$} & $\mathrm{I}$ & adult & $3(\mathrm{IOBC})$ & & & & & \multirow{2}{*}{$\begin{array}{l}\text { Unpublished } \\
\text { data }\end{array}$} \\
\hline & & & & $\mathrm{R}$ & olive leaves & $4(\mathrm{IOBC})$ & & & & & \\
\hline \multirow{2}{*}{ lambda-Cyhalothrin } & \multirow{2}{*}{$3 \mathrm{~A}$} & \multirow{2}{*}{ Karate $^{\circledR}$, ICI } & \multirow{2}{*}{$0.0038 \%$ a.i. } & $\mathrm{R}$ & glass & $4(\mathrm{IOBC})$ & & & & & \multirow{2}{*}{ [64] } \\
\hline & & & & $\mathrm{T}$ & parasitized pupa & & 1 (IOBC) & & & & \\
\hline \multirow{3}{*}{ alpha-Cypermethrin } & \multirow{3}{*}{$3 \mathrm{~A}$} & \multirow{3}{*}{ Fastac $^{\circledR}$, Shell } & \multirow{3}{*}{$0.004 \%$ a.i. } & $\mathrm{R}$ & glass & $\begin{array}{c}0.013 \\
(0.012-0.014) \\
\left(\mathrm{LT}_{50}\right) \\
\end{array}$ & & & & & [68] \\
\hline & & & & $\mathrm{R}$ & glass & $4(\mathrm{IOBC})$ & & & & & \multirow{2}{*}{ [64] } \\
\hline & & & & $\mathrm{T}$ & parasitized pupa & & 1 (IOBC) & & & & \\
\hline \multirow{2}{*}{ zeta-Cypermethrin } & \multirow{2}{*}{$3 \mathrm{~A}$} & \multirow{2}{*}{ Fury ${ }^{\circledR}$, FMC } & \multirow{2}{*}{$10 \mu \mathrm{L} / 100 \mathrm{~mL}$} & I & adult & $2(\mathrm{IOBC})$ & & & & & \multirow{2}{*}{$\begin{array}{l}\text { Unpublished } \\
\text { data }\end{array}$} \\
\hline & & & & $\mathrm{R}$ & olive leaves & $4(\mathrm{IOBC})$ & & & & & \\
\hline \multirow{2}{*}{ Deltamethrin } & \multirow{2}{*}{$3 \mathrm{~A}$} & \multirow{2}{*}{ Decis Evo ${ }^{\circledR}$, Bayer } & \multirow{2}{*}{$50 \mu \mathrm{L} / 100 \mathrm{~mL}$} & $\mathrm{I}$ & adult & $4(\mathrm{IOBC})$ & & & & & \multirow{2}{*}{$\begin{array}{l}\text { Unpublished } \\
\text { data }\end{array}$} \\
\hline & & & & $\mathrm{R}$ & olive leaves & $4(\mathrm{IOBC})$ & & & & & \\
\hline \multirow{6}{*}{ Imidacloprid } & \multirow{6}{*}{$4 \mathrm{~A}$} & $\begin{array}{l}\text { Confidor }^{\circledR}, \text { Bayer + } \\
\text { Biocebo }^{\circledR}, \text { Bioibérica }\end{array}$ & $\begin{array}{l}150 \mathrm{mg} \text { a.i./L }+1500 \mathrm{mg} \text { a.i./L } \\
\text { (bait spray) }\end{array}$ & $\mathrm{R}$ & olive leaves & $\begin{array}{c}8.0 \pm 2.0 \% \\
\left(0 \mathrm{DAT}^{4}\right) \\
1(\mathrm{IOBC})\end{array}$ & & & $35.9 \pm 4.8$ & & \multirow{2}{*}{ [78] } \\
\hline & & Confidor $^{\circledR}$, Bayer & 150 mg a.i./L (cover spray) & $\mathrm{R}$ & olive leaves & $\begin{array}{c}0.0 \pm 0.0 \% \\
(0 \mathrm{DAT}) \\
1 \text { (IOBC) }\end{array}$ & & & $55.4 \pm 4.8$ & & \\
\hline & & Confidor $^{\circledR}$, Bayer + & $\begin{array}{c}150 \mathrm{mg} \text { a.i./L }+2250 \mathrm{mg} \text { a.i. } / \mathrm{L} \\
\text { (bait spray) }\end{array}$ & $\mathrm{R}$ & olive leaves & $\begin{array}{c}8.0 \pm 3.7 \% \\
(0 \mathrm{DAT}) ; \\
1 \text { (IOBC) }\end{array}$ & & $74.0 \pm 5.0$ & $77.1 \pm 4.5$ & & \\
\hline & & Biocebo $^{\circledR}$, Bioibérica & $\begin{array}{c}1500 \text { mg a.i./L + } 2250 \text { mg a.i./L } \\
\text { (bait spray) }\end{array}$ & $\mathrm{R}$ & olive leaves & $\begin{array}{c}0.0 \pm 0.0 \% \\
\text { (0 DAT); } \\
1 \text { (IOBC) }\end{array}$ & & $72.4 \pm 5.4$ & $74.6 \pm 5.5$ & & [79] \\
\hline & & Confidor $^{\circledR}$, Bayer & $150 \mathrm{mg}$ a.i./L (cover spray) & $\mathrm{R}$ & glass & $\begin{array}{l}75.0 \pm 9.2 \% \\
3(\mathrm{IOBC})\end{array}$ & & & & & \\
\hline & & & & Sf & small olive tree & $\begin{array}{c}15.6 \pm 8.9 \% \\
3(\mathrm{IOBC})\end{array}$ & & $38.1 \pm 2.7$ & $32.5 \pm 13.1$ & & \\
\hline
\end{tabular}


Table 1. Cont.

\begin{tabular}{|c|c|c|c|c|c|c|c|c|c|c|c|}
\hline Active Ingredient (a.i.) & $\operatorname{MoA}^{1}$ & Trademark & Concentration & Exposure $^{2}$ & Substrate & Mortality $^{3}$ & Emergence $^{5}$ & $\begin{array}{c}\text { Attacked } \\
\text { Hosts }(\%)^{6}\end{array}$ & $\begin{array}{c}\text { Progeny } \\
(\%)\end{array}$ & $\begin{array}{l}\text { Longevity } \\
\text { (Days) }\end{array}$ & Reference \\
\hline \multirow{4}{*}{ Spinosad } & \multirow{4}{*}{5} & \multirow{3}{*}{$\begin{array}{l}\text { Tracer }^{\circledR}, \text { Dow } \\
\text { Agrosciences }\end{array}$} & \multirow{3}{*}{$120 \mathrm{mg}$ a.i. $/ \mathrm{L}$} & I & water & $4(\mathrm{IOBC})$ & & & & $0.5 \pm 0.1$ & \multirow{3}{*}[83]{} \\
\hline & & & & $\mathrm{R}$ & glass & $4(\mathrm{IOBC})$ & & & & $0.2 \pm 0.4$ & \\
\hline & & & & $\mathrm{T}$ & adult & $4(\mathrm{IOBC})$ & & & & $0.0 \pm 0.0$ & \\
\hline & & $\begin{array}{l}\text { Spintor-Cebo }{ }^{\circledR}, \text { Dow } \\
\text { Agrosciences }\end{array}$ & $20 \mathrm{mg}$ a.i.//L (bait spray) & $\mathrm{R}$ & olive leaves & $\begin{array}{c}4.0 \pm 2.5 \% \\
(0 \mathrm{DAT}) \\
1 \text { (IOBC) }\end{array}$ & & & $30.7 \pm 5.1$ & & {$[78]$} \\
\hline \multirow{4}{*}{ Fenoxycarb } & \multirow{4}{*}{$7 \mathrm{~B}$} & \multirow{4}{*}{ Insegar ${ }^{\circledR}$, Maag } & \multirow{2}{*}{$0.15 \%$ a.i. } & $\mathrm{R}$ & glass & 1 (IOBC) & & & & & \multirow{2}{*}{ [64] } \\
\hline & & & & $\mathrm{T}$ & parasitized pupa & 1 (IOBC) & & & & & \\
\hline & & & $5 \mathrm{~g}$ a.i. $/ \mathrm{kg}$ diet & Th & $\begin{array}{c}\text { larvae of } \\
\text { Ceratitis capitata }\end{array}$ & & $43.9 \pm 4.7 \%$ & & & $29.2 \pm 2.1$ & \multirow{2}{*}{ [87] } \\
\hline & & & $50 \mathrm{~g}$ a.i. $/ \mathrm{kg}$ diet & Th & $\begin{array}{l}\text { larvae of } \\
\text { C. capitata }\end{array}$ & & $40.1 \pm 4.6 \%$ & & & $29.8 \pm 2.6$ & \\
\hline \multirow{2}{*}{ Pyriproxyfen } & \multirow{2}{*}{$7 C$} & \multirow{2}{*}{ Juvinal ${ }^{\circledR}$, Kenogard } & 50 g i.a./ha & I & adult & $\begin{array}{c}46.6 \% \\
\text { (0 DAT); } \\
2 \text { (IOBC) }\end{array}$ & & & & & \multirow{2}{*}{ [90] } \\
\hline & & & 75 g i.a./ha & I & adult & $\begin{array}{c}53.8 \% \\
\text { (0 DAT); } \\
2 \text { (IOBC) }\end{array}$ & & & & & \\
\hline \multirow{2}{*}{ Diflubenzuron } & \multirow{2}{*}{15} & & $0.02 \mathrm{~g}$ a.i. $/ \mathrm{kg}$ diet & Th & $\begin{array}{l}\text { larvae of } \\
\text { C. capitata }\end{array}$ & & $44.2 \pm 1.6 \%$ & & & $28.5 \pm 1.6$ & \multirow{2}{*}{ [87] } \\
\hline & & & $0.2 \mathrm{~g}$ a.i. $/ \mathrm{kg}$ diet & Th & $\begin{array}{l}\text { larvae of } \\
\text { C. capitata }\end{array}$ & & $3.3 \pm 1.4 \%$ & & & & \\
\hline \multirow{5}{*}{ Tebufenozide } & \multirow{5}{*}{18} & \multirow{5}{*}{$\operatorname{Mimic}^{\circledR}$, Certis } & $0.6 \mathrm{~g}$ a.i. $/ \mathrm{kg}$ diet & Th & $\begin{array}{l}\text { larvae of } \\
\text { C. capitata }\end{array}$ & & $40.8 \pm 6.3 \%$ & & & $30.7 \pm 1.7$ & \multirow{2}{*}{ [87] } \\
\hline & & & $6 \mathrm{~g}$ a.i. $/ \mathrm{kg}$ diet & Th & $\begin{array}{l}\text { larvae of } \\
\text { C. capitata }\end{array}$ & & $26.5 \pm 8.4 \%$ & & & $31.3 \pm 2.0$ & \\
\hline & & & \multirow{3}{*}{$180 \mathrm{mg}$ a.i. $/ \mathrm{L}$} & $\mathrm{I}$ & water & 1 (IOBC) & & $91.3 \pm 3.1$ & $70.0 \pm 5.4$ & $26.3 \pm 0.4$ & \multirow{3}{*}{ [83] } \\
\hline & & & & $\mathrm{R}$ & glass & 1 (IOBC) & & $88.8 \pm 2.0$ & $71.2 \pm 2.9$ & $22.2 \pm 1.1$ & \\
\hline & & & & $\mathrm{T}$ & adult & 1 (IOBC) & & $88.3 \pm 2.2$ & $73.9 \pm 3.4$ & $27.8 \pm 1.0$ & \\
\hline
\end{tabular}


Table 1. Cont.

\begin{tabular}{|c|c|c|c|c|c|c|c|c|c|c|c|}
\hline Active Ingredient (a.i.) & $\operatorname{MoA}^{1}$ & Trademark & Concentration & Exposure $^{2}$ & Substrate & Mortality $^{3}$ & Emergence $^{5}$ & $\begin{array}{c}\text { Attacked } \\
\text { Hosts }(\%)^{6}\end{array}$ & $\begin{array}{c}\text { Progeny } \\
(\%)\end{array}$ & $\begin{array}{c}\text { Longevity } \\
\text { (Days) }\end{array}$ & Reference \\
\hline \multirow{5}{*}{ Azadirachtin } & \multirow{5}{*}{ unknown } & \multirow{5}{*}{ Align $^{\circledR}$, Sipcam } & $0.015 \mathrm{~g}$ a.i. $/ \mathrm{kg}$ diet & Th & $\begin{array}{l}\text { larvae of } \\
\text { C. capitata }\end{array}$ & & $27.3 \pm 4.0 \%$ & & & $32.0 \pm 2.0$ & \multirow{2}{*}{ [87] } \\
\hline & & & $0.15 \mathrm{~g}$ a.i. $/ \mathrm{kg}$ diet & Th & $\begin{array}{l}\text { larvae of } \\
\text { C. capitata }\end{array}$ & & $0.0 \pm 0.0 \%$ & & & & \\
\hline & & & \multirow{3}{*}{$48 \mathrm{mg}$ a.i./L } & I & water & $3(\mathrm{IOBC})$ & & $64.1 \pm 8.2$ & $43.9 \pm 4.8$ & $4.9 \pm 0.1$ & \multirow{3}{*}{ [83] } \\
\hline & & & & $\mathrm{R}$ & glass & $2(\mathrm{IOBC})$ & & $86.4 \pm 2.8$ & $43.7 \pm 5.0$ & $21.2 \pm 1.8$ & \\
\hline & & & & $\mathrm{T}$ & adult & $1(\mathrm{IOBC})$ & & $96.5 \pm 1.7$ & $71.5 \pm 4.7$ & $28.8 \pm 0.4$ & \\
\hline \multirow{6}{*}{ Kaolin } & \multirow{6}{*}{ unknown } & \multirow{6}{*}{ Surround ${ }^{\circledR}$, Basf } & $4.75 \mathrm{~kg} / \mathrm{hL}$ & $\mathrm{R}$ & olive leaves & $\begin{array}{c}2.0 \pm 2.0 \% \\
(0 \mathrm{DAT}) \\
1 \text { (IOBC) }\end{array}$ & & & $54.2 \pm 9.0$ & & [77] \\
\hline & & & $5 \mathrm{~kg} / \mathrm{hL}$ & $\mathrm{R}$ & glass & 1 (IOBC) & & 1 (IOBC) & 2 (IOBC) & & [94] \\
\hline & & & \multirow{4}{*}{$5 \mathrm{~kg} / \mathrm{hL}$} & $\mathrm{R}$ & glass & $\begin{array}{c}0.0 \pm 0.0 \% \\
(0 \mathrm{DAT}) \\
1 \text { (IOBC) }\end{array}$ & & $97.7 \pm 1.3$ & $65.0 \pm 3.9$ & & \multirow{4}{*}{ [26] } \\
\hline & & & & $\mathrm{T}$ & parasitized pupa & & $66.5 \pm 6.6 \%$ & $96.4 \pm 1.5$ & $38.6 \pm 8.3$ & & \\
\hline & & & & $\mathrm{R}$ & olive leaves & & & $89.4 \pm 2.2$ & $70.8 \pm 1.4$ & & \\
\hline & & & & Sf & olive tree & $\begin{array}{c}1.1 \pm 1.1 \% \\
(0 \mathrm{DAT}) \\
1 \text { (IOBC) }\end{array}$ & & $94.3 \pm 2.7$ & $45.2 \pm 4.3$ & & \\
\hline
\end{tabular}

${ }^{1}$ IRAC Mode of Action (MoA) classification: 1B: Organophosphates (nerve action); 3A: Pyrethrins (nerve action); 4A: Neonicotinoids (nerve action); 5: Spinosins (Nerve action);
7: Juvenile hormone mimics (growth regulation); 15: Inhibitors of chitin biosynthesis type 0 (Benzoylureas); 18: Ecdysone receptor agonists (Diacylhydrazines); Unknown: Compounds of unknown or uncertain MoA [63]. ${ }^{2}$ Intake route: R: Residual contact; T: Topical; I: Ingestion; Sf: Semifield; Th: Treated host. ${ }^{3}$ Mortality is given as category of International Organization for the Biological Control (IOBC), $\mathrm{LT}_{50}$ (lethal time that kills $50 \%$ of the treated insects), or percentage. ${ }^{4}$ DAT: days after treatment (residue age). ${ }^{5}$ Emergence is given as category of IOBC or percentage. ${ }^{6}$ Pupae without fly emergence. 


\subsection{Fungicides}

Information on the side effects of fungicides on P. concolor is scarce. The oximino-acetate trifloxystrobin (group C3) [101] did not cause any deleterious effect on P. concolor mortality, female activity and progeny (Table 2). Triazoles difenoconazole, penconazole and tebuconazole (group G1) [101] were slightly or moderately harmful to females exposed to a fresh residue on glass surface, but harmless when the parasitized C. capitata pupae were treated (Table 2) [64]. Cyproconazole was harmless even at glass residual contact tests (Table 2) [64]. Difenoconazole and tebuconazole were harmless or slightly harmful in ingestion tests, respectively, harmless in olive leave residual contact tests, and had no sublethal effects on female activity or progeny (Table 2).

Copper-based fungicides (group M01) [101], copper oxychloride, cuprous oxide and Bordeaux mixture, a mixture of copper(II) sulphate $\left(\mathrm{CuSO}_{4}\right)$ and slaked lime $\left(\mathrm{Ca}(\mathrm{OH})_{2}\right)$, were slightly harmful or harmful to females exposed to glass residual contact, but harmless when the parasitized pupae were treated (Table 2) [64]. Conversely, other formulations of copper oxychloride and Bordeaux mixture did not cause any deleterious effect on mortality neither sublethal effects on progeny in residual contact and semi-field experiments (Table 2) [26]. Similarly, copper oxychloride was completely safe to braconids A. gifuensis and A. colemani [65,72]. In this context, copper-based compounds can be considered compatible with $P$. concolor.

Sulfur (group M02) [101] had negative effects to $P$. concolor survival in glass residual contact tests, but not on parasitized pupae (Table 2) [64]. There is evidence that sulfur negatively impacts natural enemies, namely parasitoids [54,102]. Mancozeb (group M03) [101], its mixture with copper sulphate, and dithianon (group M09) [101] were completely harmless to P. concolor (Table 2) [64]. Literature indicates that mancozeb is not toxic to Anagyrus sp. near pseudococci Girault and Coccidoxenoides perminutus Timberlake (Hymenoptera: Encyrtidae) [103]. Dodine (group U12) [101] was harmful in ingestion tests but harmless in olive leave contact tests (Table 2).

Another important aspect to consider is that fungicides can reduce the diversity and richness of nectar microorganisms (fungus and bacteria) in flowers, which decreases nectar quality, sugar concentration or $\mathrm{pH}$, having consequences for the feeding of natural enemies [104]. Therefore, consequences of the application of fungicides on parasitoids should be further studied. 
Table 2. Toxicity of fungicides, currently registered in the European Union, tested on Psyttalia concolor adults.

\begin{tabular}{|c|c|c|c|c|c|c|c|c|c|c|}
\hline Active Ingredient (a.i.) & $\operatorname{MoA}^{1}$ & Trademark & Concentration & Exposure $^{2}$ & Substrate & Mortality $^{3}$ & $\begin{array}{c}\text { Emergence } \\
(\%)\end{array}$ & $\begin{array}{c}\text { Attacked } \\
{\text { hosts }(\%)^{5}}^{5}\end{array}$ & $\begin{array}{c}\text { Progeny } \\
(\%)\end{array}$ & Reference \\
\hline \multirow{2}{*}{ Trifloxystrobin } & \multirow{2}{*}{ C3 } & \multirow{2}{*}{ Flint ${ }^{\circledR}$, Bayer } & \multirow{2}{*}{$10 \mathrm{mg} / 100 \mathrm{~mL}$} & $\mathrm{I}$ & adult & $1(\mathrm{IOBC})$ & & $99.8 \pm 0.3$ & $56.7 \pm 3.5$ & \multirow{2}{*}{ Unpublished data } \\
\hline & & & & $\mathrm{R}$ & olive leaves & 1 (IOBC) & & $99.8 \pm 0.3$ & $52.2 \pm 3.2$ & \\
\hline Cyproconazole & G1 & Alto ${ }^{\circledR}$, Sandoz & $0.0186 \%$ a.i. & $\mathrm{R}$ & glass & 1 (IOBC) & & & & {$[64]$} \\
\hline \multirow{4}{*}{ Difenoconazole } & \multirow{4}{*}{ G1 } & \multirow{4}{*}{ Score $^{\circledR}$, Ciba Geigy } & \multirow{2}{*}{$0.125 \%$ a.i. } & $\mathrm{R}$ & glass & $2(\mathrm{IOBC})$ & & & & \multirow{2}{*}{ [64] } \\
\hline & & & & $\mathrm{T}$ & parasitized pupa & 1 (IOBC) & & & & \\
\hline & & & \multirow{2}{*}{$0.06 \mathrm{~mL} / 100 \mathrm{~mL}$} & I & adult & 1 (IOBC) & & $99.8 \pm 0.3$ & $52.1 \pm 4.6$ & \multirow{2}{*}{ Unpublished data } \\
\hline & & & & $\mathrm{R}$ & olive leaves & 1 (IOBC) & & $100 \pm 0.0$ & $52.8 \pm 1.6$ & \\
\hline \multirow{2}{*}{ Penconazole } & \multirow{2}{*}{ G1 } & \multirow{2}{*}{ Omnex ${ }^{\circledR}$, Ciba Geigy } & \multirow{2}{*}{$0.0025 \%$ a.i. } & $\mathrm{R}$ & glass & 2 (IOBC) & & & & \multirow{2}{*}{ [64] } \\
\hline & & & & $\mathrm{T}$ & parasitized pupa & 1 (IOBC) & & & & \\
\hline \multirow{4}{*}{ Tebuconazole } & \multirow{4}{*}{ G1 } & \multirow{4}{*}{ Folicur ${ }^{\circledR}$, Bayer } & \multirow{2}{*}{ 0.0938\% a.i. } & $\mathrm{R}$ & glass & $3(\mathrm{IOBC})$ & & & & \multirow{2}{*}{ [64] } \\
\hline & & & & $\mathrm{T}$ & parasitized pupa & 1 (IOBC) & & & & \\
\hline & & & \multirow{2}{*}{$0.06 \mathrm{~mL} / 100 \mathrm{~mL}$} & I & adult & 2 (IOBC) & & & & \multirow{2}{*}{ Unpublished data } \\
\hline & & & & $\mathrm{R}$ & olive leaves & 1 (IOBC) & & $99.3 \pm 0.8$ & $51.1 \pm 2.3$ & \\
\hline \multirow{6}{*}{ Copper oxychloride } & \multirow{6}{*}{ M01 } & \multirow{2}{*}{ Cupravit $^{\circledR}$, Bayer } & \multirow{2}{*}{ 0.196\% a.i. } & $\mathrm{R}$ & glass & 4 (IOBC) & & & & \multirow{2}{*}{ [64] } \\
\hline & & & & $\mathrm{T}$ & parasitized pupa & 1 (IOBC) & & & & \\
\hline & & \multirow{4}{*}{ ZZ-cuprocol ${ }^{\circledR}$, Syngenta Agro } & \multirow{4}{*}{$250 \mathrm{~mL} / \mathrm{hL}$} & $\mathrm{R}$ & glass & $\begin{array}{l}0.0 \pm 0.0 \% \\
\left(0 \mathrm{DAT}^{4}\right) ; \\
1(\mathrm{IOBC})\end{array}$ & & $92.2 \pm 5.8$ & $52.1 \pm 4.4$ & \multirow{4}{*}{ [26] } \\
\hline & & & & $\mathrm{T}$ & parasitized pupa & & $63.6 \pm 3.65$ & $98.8 \pm 0.3$ & $30.0 \pm 13.9$ & \\
\hline & & & & $\mathrm{R}$ & olive leaves & & & $98.3 \pm 0.8$ & $78.6 \pm 3.6$ & \\
\hline & & & & Sf & olive tree & $\begin{array}{c}1.1 \pm 1.1 \% \\
(0 \mathrm{DAT}) \\
1 \text { (IOBC) }\end{array}$ & & $93.3 \pm 3.9$ & $45.3 \pm 4.1$ & \\
\hline \multirow{2}{*}{ Cuprous oxide } & M01 & Oxiram ${ }^{\circledR}$ Ciba-Geigy & $0.3 \%$ a.i. & $\mathrm{R}$ & glass & $3(\mathrm{IOBC})$ & & & & [64] \\
\hline & & & & $\mathrm{T}$ & parasitized pupa & 1 (IOBC) & & & & \\
\hline
\end{tabular}


Table 2. Cont.

\begin{tabular}{|c|c|c|c|c|c|c|c|c|c|c|}
\hline Active Ingredient (a.i.) & $\mathrm{MoA}^{1}$ & Trademark & Concentration & Exposure $^{2}$ & Substrate & Mortality $^{3}$ & $\begin{array}{c}\text { Emergence } \\
(\%)\end{array}$ & $\begin{array}{c}\text { Attacked } \\
\text { hosts (\%) }^{5}\end{array}$ & $\begin{array}{c}\text { Progeny } \\
(\%)\end{array}$ & Reference \\
\hline \multirow{6}{*}{ Bordeaux mixture } & \multirow{6}{*}{ M01 } & \multirow{2}{*}{ C. Bord Vallès ${ }^{\circledR}$, I. Q. Vallès } & \multirow{2}{*}{$0.2 \%$ a.i. } & $\mathrm{R}$ & glass & 2 (IOBC) & & & & \multirow{2}{*}{ [64] } \\
\hline & & & & $\mathrm{T}$ & parasitized pupa & 1 (IOBC) & & & & \\
\hline & & \multirow{4}{*}{ Poltiglia ${ }^{\circledR}$, Manica SPA } & \multirow{4}{*}{$1 \mathrm{~kg} / \mathrm{hL}$} & $\mathrm{R}$ & glass & $\begin{array}{c}0.0 \pm 0.0 \% \\
(0 \mathrm{DAT}) \\
1(\mathrm{IOBC})\end{array}$ & & $91.8 \pm 3.7$ & $60.2 \pm 4.8$ & \multirow{4}{*}{ [26] } \\
\hline & & & & $\mathrm{T}$ & parasitized pupa & & $64.8 \pm 4.3$ & $98.7 \pm 0.9$ & $37.9 \pm 10.4$ & \\
\hline & & & & $\mathrm{R}$ & olive leaves & & & $94.5 \pm 1.1$ & $72.3 \pm 4.9$ & \\
\hline & & & & Sf & olive tree & $\begin{array}{c}3.3 \pm 1.9 \% \\
(0 \mathrm{DAT}) \\
1(\mathrm{IOBC})\end{array}$ & & $90.2 \pm 6.5$ & $54.0 \pm 3.5$ & \\
\hline \multirow{2}{*}{ Sulfur } & \multirow{2}{*}{ M02 } & \multirow{2}{*}{ Microtox $^{\circledR}$, Agrocros } & \multirow{2}{*}{$0.432 \%$ a.i. } & $\mathrm{R}$ & glass & 3 (IOBC) & & & & \multirow{2}{*}{ [64] } \\
\hline & & & & $\mathrm{T}$ & parasitized pupa & 1 (IOBC) & & & & \\
\hline Mancozeb & M03 & Dithane M- $45^{\circledR}$, Rohm and Haas & $0.208 \%$ a.i. & $\mathrm{R}$ & glass & 1 (IOBC) & & & & [64] \\
\hline $\begin{array}{l}\text { Mancozeb + copper } \\
\text { sulphate }\end{array}$ & $\begin{array}{l}\text { M03, } \\
\text { M01 }\end{array}$ & $\begin{array}{c}\text { Cuprodithane }^{\circledR}+\text { Fuerte }^{\circledR}, \\
\text { Rohm and Haas }\end{array}$ & $0.048 \%$ a.i. $+0.144 \%$ a.i. & $\mathrm{R}$ & glass & 1 (IOBC) & & & & [64] \\
\hline Dithianon & M09 & Delan $75^{\circledR}$, Shell & $0.075 \%$ a.i. & $\mathrm{R}$ & glass & 1 (IOBC) & & & & [64] \\
\hline \multirow{2}{*}{ Dodine } & \multirow{2}{*}{ U12 } & \multirow{2}{*}{ Syllit Flow ${ }^{\circledR}$, Kenogard } & \multirow{2}{*}{$0.225 \mathrm{~mL} / 100 \mathrm{~mL}$} & I & adult & 3 (IOBC) & & & & \multirow{2}{*}{ Unpublished data } \\
\hline & & & & $\mathrm{R}$ & olive leaves & 1 (IOBC) & & $99.8 \pm 0.3$ & $51.4 \pm 2.0$ & \\
\hline
\end{tabular}

${ }^{1}$ FRAC Mode of Action (MoA) classification: G1: Sterol biosynthesis in membranes (Triazoles); M01: Chemicals with multi-site activity (Inorganic copper); M02: Chemicals with multi-site activity (Inorganic sulfur); M03: Chemicals with multi-site activity (Dithio-carbamates and relatives); M09: Chemicals with multi-site activity (Quinones); U: Unknown mode of action [101] ${ }^{2}$ Intake route: I: Ingestion; R: Residual contact; T: Topical; Sf: Semifield. ${ }^{3}$ Mortality is given as category of IOBC or percentage. ${ }^{4}$ DAT: days after treatment (residue age). ${ }^{5}$ Pupae without fly emergence. 


\subsection{Herbicides}

From an integrated weed control strategy perspective, it is important to select herbicides compatible with natural enemies. The only study available on the effects of herbicides on P. concolor adult females found that the pyridyloxy-carboxylate fluroxypyr (group 4) [105] and the triazone metamitron (group 5) [105] were slightly harmful in residual contact assays, but harmless when the parasitized pupa was treated (Table 3) [64]. The phosphonate glyphosate (group 9); [105] was slightly harmful in ingestion tests but safe in olive leave residual contact tests, without any deleterious effect on female activity or progeny (Table 3). The benzofurane ethofumesate (group 15) [105] was completely safe, even when tested at glass surface (Table 3) [64]. In conclusion, although these herbicides did not prove to be toxic to $P$. concolor, literature is scarce and further studies are needed to understand the persistence of herbicides on olive groves and, consequently, the real implications on parasitoids. As mentioned for fungicides, some herbicides can also decrease pollen production and viability [50]. Besides that, the indiscriminate application of herbicides can reduce floral community diversity, removes refuges for natural enemies or plants with alternative hosts [106]. Herbicide studies have been traditionally neglected, especially in the case of pre-emergence herbicides, due to its uncommon presence in the exposure methodology proposed by IOBC. Nevertheless, from a broader perspective, all herbicides can indirectly affect $P$. concolor population as they limit the growth of plants providing food or shelter. In addition, post-emergence herbicides might potentially reduce the survival and reproduction of P. concolor adults. 
Table 3. Toxicity of herbicides, currently registered in the European Union, tested on Psyttalia concolor adults.

\begin{tabular}{|c|c|c|c|c|c|c|c|c|c|}
\hline Active Ingredient (a.i.) & $\operatorname{MoA}^{1}$ & Trademark & Concentration & Exposure $^{2}$ & Substrate & Mortality $^{3}$ & Attacked Hosts (\%) ${ }^{4}$ & Progeny $(\%)$ & Reference \\
\hline \multirow{2}{*}{ Fluroxypyr } & \multirow{2}{*}{4} & \multirow{2}{*}{ Starane $180^{\circledR}$, Dow Elanco } & \multirow{2}{*}{$0.09 \%$ a.i. } & $\mathrm{R}$ & glass & $2(\mathrm{IOBC})$ & & & \multirow{2}{*}{ [64] } \\
\hline & & & & $\mathrm{T}$ & parasitized pupa & 1 (IOBC) & & & \\
\hline \multirow{2}{*}{ Metamitron } & \multirow{2}{*}{5} & \multirow{2}{*}{ Goltix ${ }^{\circledR}$, Bayer } & \multirow{2}{*}{$1.75 \%$ a.i. } & $\mathrm{R}$ & glass & $2(\mathrm{IOBC})$ & & & \multirow{2}{*}{ [64] } \\
\hline & & & & $\mathrm{T}$ & parasitized pupa & $1(\mathrm{IOBC})$ & & & \\
\hline \multirow{2}{*}{ Glyphosate } & \multirow{2}{*}{9} & \multirow{2}{*}{ Touchdown ${ }^{\circledR}$, Special } & \multirow{2}{*}{$0.7 \mathrm{~mL} / 100 \mathrm{~mL}$} & $\mathrm{I}$ & adult & 2 (IOBC) & & & \multirow{2}{*}{ Unpublished data } \\
\hline & & & & $\mathrm{R}$ & olive leaves & 1 (IOBC) & $99.8 \pm 0.3$ & $53.4 \pm 4.2$ & \\
\hline Ethofumesate & 15 & Tramat $500^{\circledR}$, Schering & $0.5 \%$ a.i. & $\mathrm{R}$ & glass & 1 (IOBC) & & & [64] \\
\hline
\end{tabular}

${ }^{1}$ HRAC Mode of Action (MoA) Classification: 4: Auxin mimics (Pyridyloxy-carboxylates); 5: D1 Serine 264 binders (Triazinones); 9: Inhibition of enolpyruvyl shikimate phosphate synthase; 15: Inhibition of very long-chain fatty acid synthesis (Benzofuranes) [105]. ${ }^{2}$ Intake route: R: Residual contact; T: Topical, I: Ingestion. ${ }^{3}$ Mortality is given as category of IOBC. ${ }_{4}^{4}$ Pupae without fly emergence. 


\subsection{Microbial Insecticides}

Microorganisms (bacteria, fungi, viruses, and others) have good potential as biopesticides because of their high selectivity and safety to non-target organisms [107,108]. Bacillus thuringiensis, a microbial disruptor of midgut membranes (group 11A) [63], has a relatively broad spectrum of activity against coleopterans (subspecies tenebrinonis and lentimorbus), dipterans (subspecies israelensis) and lepidopterans (subspecies kurstaki and aizawai) [109]. Subspecies kurstaki was compatible with P. concolor (Table 4) [64]. Eleven field isolates of subspecies israelensis collected in several countries, which produced significant mortality to B. oleae, were safe for P. concolor adults in ingestion tests (Table 4) [110]. Likewise, subspecies kurstaki was harmless for braconids B. nigricans and A. colemani $[72,85]$. On the other hand, subspecies kurstaki did not affect the reproductive success of Trichogramma chilonis Ishii (Hymenoptera: Trichogrammatidae) while a significant effect was observed on longevity and the time spent on host eggs patches [111].

Fungal agent Beauveria bassiana is another biopesticide used to control the olive fruit fly [112]. Beauveria bassiana did not cause any mortality on P. concolor (Table 4) [14]. However, their beneficial capacity was negatively affected via residual contact or treated host (lower progeny size), or by ingestion (fewer attacked hosts). Also, P. concolor emergence decreased by $50 \%$ compared to control when the fungal treatment was applied to parasitized pupae (Table 4) [113]. Although some studies evidenced that some predators and parasitoids are susceptible in laboratory conditions, impact is minimum in the field [114]. On the other hand, Metarhizium anisopliae did not posed any adverse effect on the emergence of P. concolor (Table 4) [115]. Entomopathogenic nematode species Heterorhabditis megidis Poinar, Steinernema feltiae Filipjev and H. bacteriophora are not entirely safe for P. concolor. Although they did not cause direct mortality to $P$. concolor, they decreased the progeny size when females parasitized on treated larvae, compared to control, but significant reductions were only observed for S. feltiae (Table 4) [14].

For a safer use of microorganism-based biopesticides that can be used concomitantly with P. concolor, more studies about side effects are needed. Also, microorganisms present in the field should be further studied as landscape structure, crucial for the establishment of arthropod community, constitutes an additional reservoir of genetic diversity with potential for pest management $[39,40]$.

\subsection{Botanical Compounds}

Plant extracts and oils might be also used as alternative to conventional insecticides. Extracts of Trichilia havanensis Jacq. (Meliaceae) seeds, F12 limonoids (azadirone) and F18 (1.7 + 3.7-di-O-acetilhavanensin), and Teucrium viscidum (Lamiaceae) seeds, M1 (Teucjaponic B) and M9 (Teucvin), did not cause mortality on P. concolor, neither reduced the beneficial capacity in topical or ingestion laboratory conditions (Table 4) $[13,116]$. Similar results were obtained when the olive fruit was treated with Peganum harmala L. (Zygophllaceae) seed extract. There was no difference in the emergence of $P$. concolor between treated and untreated fruit, wasps actively searched for hosts and parasitoid mortality was not registered (Table 4) [117]. Conversely, Melaleuca alternifolia (Myrtaceae) essential oil was slightly to moderately harmful to P. concolor, although it was considered more toxic for the Mediterranean fruit fly, C. capitata, than for the parasitoid (Table 4) [118]. Finally, the mixture of essential oils of Cymbopogon citratus (DC.) Stapf (Poaceae), Cedrus atlantica (Endl.) Manetti ex Carriére (Pinaceae) and Corymbia citriodora (Hook.) K.D. Hill and L.A.S. Johnson (Myrtaceae) was harmless to P. concolor (Table 4) [119].

Despite the limitations of botanical compounds on pest control, some authors have reported a certain degree of efficacy of these products on pests B. oleae and C. capitata [117-119]. Thus, there is a need to extend the research and use of these promising compounds within IPM programs of target pests. Overall, microbial and botanical pesticides are harmless to P. concolor and should be used to increase the sustainability of agricultural systems as these products have environmental safety, target-specificity, efficacy and biodegradability [120]. 
Table 4. Toxicity of microbial insecticides and botanical compounds tested on Psyttalia concolor adults.

\begin{tabular}{|c|c|c|c|c|c|c|c|c|c|c|}
\hline Active Ingredient (a.i.) & $\operatorname{MoA}^{1}$ & Trademark & Concentration & Exposure $^{2}$ & Substrate & Mortality $^{3}$ & Emergence $^{6}$ & $\begin{array}{c}\text { Attacked } \\
\text { Hosts (\%) }{ }^{7}\end{array}$ & Progeny (\%) & Reference \\
\hline \multicolumn{11}{|c|}{ Entomopathogenic bacteria } \\
\hline \multirow{3}{*}{ Bacillus thuringiensis var. kurstaki } & \multirow{3}{*}{$11 \mathrm{~A}$} & \multirow{2}{*}{$\begin{array}{l}\text { Bactospeine }{ }^{\circledR}, \text { S.C. } \\
\text { Agrocrós }\end{array}$} & \multirow{2}{*}{$1.7 \times 10^{7} \mathrm{IU} / \mathrm{L}$} & $\mathrm{R}$ & glass & 1 (IOBC) & & & & \multirow{2}{*}{ [64] } \\
\hline & & & & $\mathrm{T}$ & parasitized pupa & 1 (IOBC) & & & & \\
\hline & & Delfin DG ${ }^{\circledR}$, Sandoz & $3.2 \times 10^{7} \mathrm{IU} / \mathrm{L}$ & $\mathrm{R}$ & glass & 1 (IOBC) & & & & [64] \\
\hline Bacillus thuringiensis var. israelensis & $11 \mathrm{~A}$ & Field isolates & $10^{6} \mathrm{IU} / \mathrm{L}$ & I & water & 1 (IOBC) & & & & [110] \\
\hline \multicolumn{11}{|c|}{ Entomopathogenic fungus } \\
\hline \multirow{5}{*}{ Beauveria bassiana } & \multirow{5}{*}{-} & \multirow{5}{*}{$\begin{array}{l}\text { Naturalis } L^{\circledR}, \\
\text { Agrichem }\end{array}$} & \multirow{4}{*}{$2.3 \times 10^{12}$ conidia $/ \mathrm{hL}$} & $\mathrm{R}$ & glass & $\begin{array}{l}10.8 \pm 2.0 \% \\
\left(0 \mathrm{DAT}^{5}\right)\end{array}$ & & $42.8 \pm 2.0$ & $81.2 \pm 5.5$ & \multirow{4}{*}{ [14] } \\
\hline & & & & $\mathrm{T}$ & drop in the prothorax ${ }^{4}$ & $\begin{array}{l}2.9 \pm 0.9 \% \\
(0 \mathrm{DAT})\end{array}$ & & $60.0 \pm 2.8$ & $71.7 \pm 2.8$ & \\
\hline & & & & I & water & $\begin{array}{c}3.6 \pm 1.2 \% \\
(0 \mathrm{DAT})\end{array}$ & & $31.9 \pm 3.1$ & $81.7 \pm 3.0$ & \\
\hline & & & & Th & larvae of Ceratitis capitata & & & $69.4 \pm 3.5$ & $48.6 \pm 3.4$ & \\
\hline & & & $2.3 \times 10^{10}$ conidia $/ \mathrm{hL}$ & $\mathrm{T}$ & parasitized pupa & & 2 (IOBC) & & & [113] \\
\hline Metarhizium anisopliae & - & Field isolate & $4 \times 10^{12}$ conidia/ha & $\mathrm{T}$ & parasitized pupa & & $54.2 \pm 12.6 \%$ & & & [115] \\
\hline \multicolumn{11}{|c|}{ Entomopathogenic nematodes } \\
\hline Heterorhabditis bacteriophora & - & Larvanem ${ }^{\circledR}$, Koppert & $\begin{array}{r}100 \text { infective } \\
\text { juveniles } / \mathrm{cm}^{2}\end{array}$ & Th & larvae of C. capitata & & & & $15 \%$ & [14] \\
\hline Heterorhabditis megidis & - & $\begin{array}{l}\text { Heterorhabditis } \\
\text { system }{ }^{\circledR}, \text { Biobest }\end{array}$ & $\begin{array}{l}100 \text { infective } \\
\text { juveniles } / \mathrm{cm}^{2}\end{array}$ & Th & larvae of C. capitata & & & & $20 \%$ & [14] \\
\hline Steinernema feltiae & - & $\begin{array}{c}\text { Steinernema system }{ }^{\circledR}, \\
\text { Biobest }\end{array}$ & $\begin{array}{l}100 \text { infective } \\
\text { juveniles } / \mathrm{cm}^{2}\end{array}$ & Th & larvae of C. capitata & & & & $9.5 \%$ & [14] \\
\hline \multicolumn{11}{|c|}{ Botanical compounds } \\
\hline \multirow{3}{*}{ F12 Limonoids (azadirone) } & & & \multirow{3}{*}{$1000 \mathrm{mg}$ a.i./L } & I & water & $\begin{array}{c}3.3 \pm 3.3 \% \\
(0 \mathrm{DAT}) \\
1 \text { (IOBC) }\end{array}$ & & $89.1 \pm 1.9$ & $50.2 \pm 4.5$ & \multirow{2}{*}{ [116] } \\
\hline & - & - & & $\mathrm{T}$ & drop in the prothorax ${ }^{4}$ & $\begin{array}{c}3.3 \pm 3.3 \% \\
(0 \mathrm{DAT}) \\
1 \text { (IOBC) }\end{array}$ & & $87.0 \pm 3.6$ & $51.7 \pm 3.9$ & \\
\hline & & & & I & water & $\begin{array}{c}10.0 \pm 3.3 \% \\
(0 \text { DAT })\end{array}$ & & $74.2 \pm 3.5$ & $63.6 \pm 3.4$ & [13] \\
\hline
\end{tabular}


Table 4. Cont.

\begin{tabular}{|c|c|c|c|c|c|c|c|c|c|c|}
\hline Active Ingredient (a.i.) & $\operatorname{MoA}^{1}$ & Trademark & Concentration & Exposure $^{2}$ & Substrate & Mortality $^{3}$ & Emergence $^{6}$ & $\begin{array}{c}\text { Attacked } \\
{\text { Hosts }(\%)^{7}}^{7}\end{array}$ & Progeny $(\%)$ & Reference \\
\hline \multirow{3}{*}{$\begin{array}{c}\text { F18 } \\
(1.7+3.7-d i-O-a c e t i l h a v a n e n s i n)\end{array}$} & \multirow{3}{*}{ - } & & \multirow{3}{*}{$1000 \mathrm{mg}$ a.i./L } & I & water & $\begin{array}{c}3.3 \pm 1.9 \% \\
(0 \mathrm{DAT}) \\
1 \text { (IOBC) }\end{array}$ & & $85.0 \pm 2.6$ & $48.2 \pm 5.9$ & \multirow{2}{*}{ [116] } \\
\hline & & ـ & & $\mathrm{T}$ & drop in the prothorax ${ }^{4}$ & $\begin{array}{c}6.7 \pm 2.7 \% \\
(0 \mathrm{DAT}) \\
1 \text { (IOBC) }\end{array}$ & & $85.5 \pm 3.4$ & $51.2 \pm 4.4$ & \\
\hline & & & & I & water & $\begin{array}{c}11.7 \pm 3.2 \% \\
(0 \mathrm{DAT})\end{array}$ & & $70.6 \pm 5.5$ & $57.5 \pm 6.9$ & [13] \\
\hline M1 (Teucjaponic B) & - & - & $1000 \mathrm{mg}$ a.i. $/ \mathrm{L}$ & I & water & $\begin{array}{l}11.7 \pm 1.7 \% \\
(0 \mathrm{DAT})\end{array}$ & & $64.9 \pm 5.2$ & $49.4 \pm 4.4$ & [13] \\
\hline M9 (Teucvin) & - & - & $1000 \mathrm{mg}$ a.i. $/ \mathrm{L}$ & I & water & $\begin{array}{c}6.7 \pm 3.8 \% \\
(0 \mathrm{DAT})\end{array}$ & & $66.9 \pm 6.0$ & $51.2 \pm 3.8$ & [13] \\
\hline Peganum harmala seed extract & - & - & $2 \%(w / w)$ & Th & larvae of Bactrocera oleae & 1 (IOBC) & $1(\mathrm{IOBC})$ & & & [117] \\
\hline \multirow{3}{*}{ Melaleuca alternifolia oil } & \multirow{3}{*}{-} & \multirow{3}{*}{-} & 0.1 to $2 \%(w / w)$ & I & water & $\begin{array}{l}0.639 \% \\
\left(\mathrm{LD}_{50}\right) \\
<35 \%\end{array}$ & & & & \multirow{3}{*}{ [118] } \\
\hline & & & 0.1 to $3 \mu \mathrm{L} / \mathrm{cm}^{2}$ & $\mathrm{R}$ & filter paper & $\begin{array}{l}0.147 \% \\
\left(\mathrm{LC}_{50}\right) \\
>60 \%\end{array}$ & & & & \\
\hline & & & 0.15 to $18 \mu \mathrm{L} / \mathrm{L}$ air & $\mathrm{F}$ & filter paper & $\begin{array}{l}9.348 \% \\
\left(\mathrm{LC}_{50}\right) ; \\
>50 \%\end{array}$ & & & & \\
\hline $\begin{array}{l}\text { Cymbopogon citratus, Cedrus } \\
\text { atlantica, Corymbia citriodora oils }\end{array}$ & - & - & $4.8 \%(w / w)$ & Sf & orange tree & $\begin{array}{c}0.0 \pm 0.0 \% \\
(0 \mathrm{DAT}) \\
1 \text { (IOBC) }\end{array}$ & & & & [119] \\
\hline
\end{tabular}

${ }^{1}$ IRAC Mode of Action (MoA) classification: 11A: Microbial disruptors of insect midgut membranes [63]. ${ }^{2}$ Intake route: R: Residual contact; T: Topical; I: Ingestion; Th: Treated host;

F: Fumigation; Sf: Semifield. ${ }^{3}$ Mortality is given as category of IOBC, percentage, or $\mathrm{LD}_{50} / \mathrm{LC}_{50}$ (lethal dose/lethal concentration that kills $50 \%$ of the treated insects). ${ }^{4} 0.5 \mathrm{microliters/insect.}$

${ }^{5}$ DAT: days after treatment (residue age). ${ }^{6}$ Emergence is given as category of IOBC or percentage. ${ }^{7}$ Pupae without fly emergence. 


\section{Conclusions}

- Insecticides cause more negative effects on P. concolor survival and reproduction than fungicides, herbicides or biopesticides. Neurotoxic organophosphates and pyrethroids are the most toxic insecticides to $P$. concolor adults, however they are usually harmless when applied in host pupae stage, the most protected life stage. IGRs and kaolin are alternative pesticides compatible with $P$. concolor, however the variety of responses urges for more research on side effects of this parasitoid.

- Despite that the impact of fungicides on P. concolor is milder, some are harmful at residual contact tests, thus negative effects due to foliar application on the olive canopy should not be ignored.

- Most studies focus on mortality and reproduction, but other sublethal effects such as longevity, learning performance, behavior, neurophysiology, physiological or immunology should also be considered.

- Most studies only consider a single compound but not the synergies of several substances applied at the same time. For example, there are no data on the joint use of fungicides and insecticides.

- Literature about laboratory studies supported with field data is very scarce, especially for herbicides, biopesticides and botanical extracts. Field ecotoxicological studies would allow the optimization of the management of parasitoids after pesticide applications, establishing the use of harmless pesticides for P. concolor as a pre-requisite for the control of B. oleae.

- The number of independent publications related to scientific ecotoxicological studies, beyond those required for the registration of plant protection products, have sharply decreased in the EU. Consequently, there is a need for updated data on the toxicity of novel substances on important natural enemies of relevant crops, such as P. concolor.

Author Contributions: Conceptualization, J.A.P., S.A.P.S., and P.M.; investigation, L.A.P. and A.C.W.; data curation, L.A.P., B.D., and P.M.; writing-original draft preparation, L.A.P., B.D., and P.M.; writing-review and editing, all authors; supervision, J.A.P., S.A.P.S., and P.M.; funding acquisition, J.A.P. and S.A.P.S. All authors have read and agreed to the published version of the manuscript.

Funding: The authors are grateful to THE FOUNDATION FOR SCIENCE AND TECHNOLOGY (FCT, Portugal), for financial support through national funds FCT/MCTES to CIMO, grant number UIDB/00690/2020. Lara A. Pinheiro is grateful to FCT for PhD studentship SFRH/BD/103998/2014. Beatriz Dáder is grateful to SPANISH MINISTRY OF SCIENCE AND INNOVATION for fellowship IJC2018-035042-I and to UNIVERSIDAD POLITÉCNICA DE MADRID for funding, grant number PINV18XFWLGK24S2US6D.

Conflicts of Interest: The authors declare no conflict of interest.

\section{References}

1. Oerke, E.C. Crop losses to pests. J. Agric. Sci. 2006, 144, 31-43. [CrossRef]

2. Casida, J.E.; Quistad, G.B. Golden age of insecticide research: Past, present, or future? Annu. Rev. Entomol. 1998, 43, 1-16. [CrossRef] [PubMed]

3. Cooper, J.; Dobson, H. The benefits of pesticides to mankind and the environment. Crop. Prot. 2007, 26, 1337-1348. [CrossRef]

4. Denholm, I.; Pickett, J.A.; Devonshire, A.L. Insecticide Resistance: From Mechanisms to Management; CABI and The Royal Society publishing: Egham, UK, 2001; ISBN 0-85199-367-2.

5. Desneux, N.; Decourtye, A.; Delpuech, J.M. The sublethal effects of pesticides on beneficial arthropods. Annu. Rev. Entomol. 2007, 52, 81-106. [CrossRef]

6. Weinzierl, R.A. Integrating pesticides with biotic and biological control for arthropod pest management. In Integrated Pest Management: Concepts, Tactics, Strategies and Case Studies; Cambridge University Press: Cambridge, UK, 2008; pp. 179-191.

7. Cloyd, R. Indirect effects of pesticides on natural enemies. In Pesticides Advances in Chemical and Botanical Pesticides; Soundararajan, R.P., Ed.; IntechOpen: London, UK, 2012; ISBN 978-953-51-0680-7. [CrossRef]

8. Ehler, L.E. Perspective. Integrated pest management (IPM): Definition, historical development and implementation, and the other IPM. Pest. Manag. Sci. 2006, 62, 787-789. [CrossRef] 
9. Stenberg, J.A. A conceptual framework for Integrated Pest Management. Trends. Plant. Sci. 2017, 22, 759-769. [CrossRef]

10. OJEU (Official Journal of the European Union). Directive 2009/128/EC of the European Parliament and of the Council of 21 October 2009, establishing a framework for Community action to achieve the sustainable use of pesticides. OJEU 2009, 209, 71-86.

11. Kenis, M.; Hurley, B.P.; Hajek, A.E.; Cock, M.J.W. Classical biological control of insect pests of trees: Facts and figures. Biol. Invasions 2017, 19, 3401-3417. [CrossRef]

12. Gentz, M.C.; Murdoch, G.; King, G.F. Tandem use of selective insecticides and natural enemies for effective, reduced-risk pest management. Biol. Control. 2010, 52, 208-215. [CrossRef]

13. Medina, P.; Budia, F.; González, M.; Rodríguez, B.; Díaz, A.; Huerta, A.; Zapata, N.; Viñuela, E. Effects of botanical insecticides on two natural enemies of importance in Spain: Chrysoperla carnea (Stephens) and Psyttalia concolor (Szépligeti). Pesticides and Beneficial Organisms. IOBC/WPRS Bull. 2005, 29, 85-93.

14. Medina, P.; Corrales, E.; González-Núñez, M.; Smagghe, G.; Viñuela, E. Effects of Beauveria bassiana, Heterorhabditis bacteriophora, $H$. megidis and Steinernema feltiae on the Mediterranean fruit fly Ceratitis capitata and the very sensitive braconid Psyttalia concolor in the lab. Pesticides and Beneficial Organisms. IOBC/WPRS Bull. 2008, 35, 113-121.

15. Garzón, A.; Medina, P.; Amor, F.; Viñuela, E.; Budia, E. Toxicity and sublethal effects of six insecticides to last instar larvae and adults of the biocontrol agents Chrysoperla carnea (Stephens) (Neuroptera: Chrysopidae) and Adalia bipunctata (L.) (Coleoptera: Coccinellidae). Chemosphere 2015, 132, 87-93. [CrossRef] [PubMed]

16. Geiger, F.; Bengtsson, J.; Berendse, F.; Weisser, W.W.; Emmerson, M.; Morales, M.B.; Ceryngier, P.; Liira, J.; Tscharntke, T.; Winqvist, C.; et al. Persistent negative effects of pesticides on biodiversity and biological control potential on European farmland. Basic Appl. Ecol. 2010, 11, 97-105. [CrossRef]

17. Daane, K.M.; Johnson, M.W. Olive fruit fly: Managing an ancient pest in modern times. Annu. Rev. Entomol. 2010, 55, 151-169. [CrossRef]

18. Malheiro, R.; Casal, S.; Baptista, P.; Pereira, J.A. Review of Bactrocera oleae (Rossi) impact in olive products: From the tree to the table. Trends Food. Sci. Technol. 2015, 44, 226-242. [CrossRef]

19. Quesada-Moraga, E.; Santiago-Álvarez, C.; Cubero-González, S.; Casado-Mármol, G.; Ariza-Fernández, A.; Yousef, M. Field evaluation of the susceptibility of mill and table olive varieties to egg-laying of olive fly. J. Appl. Entomol. 2018, 142, 765-774. [CrossRef]

20. Kampouraki, A.; Stavrakaki, M.; Karataraki, A.; Katsikogiannis, G.; Pitika, E.; Varikou, K.; Vlachaki, A.; Chrysargyris, A.; Malandraki, E.; Sidiropoulos, N.; et al. Recent evolution and operational impact of insecticide resistance in olive fruit fly Bactrocera oleae populations from Greece. J. Pest. Sci. 2018, 91, 1429-1439. [CrossRef]

21. Kalaitzaki, A.; Varikou, K.; Alexandrakis, V. Study of the effectiveness of various insecticides used for the control of Bactrocera oleae (Gmelin) (Diptera: Tephritidae) in Crete olive groves. IOBC/WPRS Bull. 2010, 53, 37-46.

22. Vontas, J.G.; Hernández-Crespo, P.; Margaritopoulos, J.T.; Ortego, F.; Feng, H.T.; Mathiopoulos, K.D.; Hsu, J.C. Insecticide resistance in tephritid flies. Pestic. Biochem. Physiol. 2011, 100, 199-205. [CrossRef]

23. Bengochea, P.; Christiaens, O.; Amor, F.; Viñuela, E.; Rouge, P.; Medina, P.; Smagghe, G. Insect growth regulators as potencial insecticides to control olive fruit fly (Bactrocera oleae Rossi): Insect toxicity bioassays and molecular docking approach. Pest. Manag. Sci. 2012, 69, 27-34. [CrossRef]

24. Sánchez-Ramos, I.; Fernández, C.E.; González-Núñez, M.; Pascual, S. Laboratory tests of insect growth regulators as bait sprays for the control of the olive fruit fly, Bactrocera oleae (Diptera: Tephritidae). Pest. Manag. Sci. 2013, 69, 520-526. [CrossRef] [PubMed]

25. Varikou, K.; Garantonakis, N.; Birouraki, A.; Ioannou, A.; Kapogia, E. Improvement of bait sprays for the control of Bactrocera oleae (Diptera: Tephritidae). Crop. Prot. 2016, 81, 1-8. [CrossRef]

26. Bengochea, P.; Budia, F.; Viñuela, E.; Medina, P. Are kaolin and copper treatments safe to the olive fruit fly parasitoid Psyttalia concolor? J. Pest. Sci. 2014, 87, 351-359. [CrossRef]

27. Vontas, J.G.; Hejazi, M.J.; Hawkes, N.J.; Cosmidis, N.; Loukas, M.; Hemingway, J. Resistance-associated point mutations of organophosphate insensitive acetylcholinesterase, in the olive fruit fly Bactrocera oleae. Insect. Mol. Biol. 2002, 11, 329-336. [CrossRef] [PubMed]

28. Kakani, E.G.; Mathiopoulos, K.D. Organophosphosphate resistance-related mutations in the acetylcholinesterase gene of Tephritidae. J. Appl. Entomol. 2008, 132, 762-771. [CrossRef] 
29. Kakani, E.G.; Zygouridis, N.E.; Tsoumani, K.T.; Seraphides, N.; Zalom, F.G.; Mathiopoulos, K.D. Spinosad resistance development in wild olive fruit fly Bactrocera oleae (Diptera: Tephritidae) populations in California. Pest. Manag. Sci. 2010, 66, 447-453. [CrossRef] [PubMed]

30. Sirjani, F.O.; Lewis, E.E.; Kaya, H.K. Evaluation of entomopathogenic nematodes against the olive fruit fly, Bactrocera oleae (Diptera: Tephritidae). Biol. Control. 2009, 48, 274-280. [CrossRef]

31. Torrini, G.; Mazza, G.; Benvenuti, C.; Roversi, P.F. Susceptibility of olive fruit fly, Bactrocera oleae (Diptera: Tephritidae) pupae to entomopathogenic nematodes. J. Plant Prot. Res. 2017, 57, 318-320. [CrossRef]

32. Arambourg, Y. Traité D'entomologie Oléicole; Conseil Oleicole International: Madrid, Spain, 1986.

33. Pinheiro, L.A.; Medina, P.; Bento, A.; Pereira, J.A.; Santos, S.A.P. Bioecology of Psyttalia concolor (Szépligeti) (Hymenoptera: Braconidae) and management strategies to increase biological control of the olive fruit fly. In Natural Enemies: Identification, Protection Strategies and Ecological Impacts; Santos, S.A.P., Ed.; Nova Science Publishers: New York, NY, USA, 2017; pp. 141-172.

34. Boccacio, L.; Petacchi, R. Landscape effects on the complex of Bactrocera oleae parasitoids and implications for conservation biological control. BioControl 2009, 54, 607-616. [CrossRef]

35. Miranda, M.A.; Miquel, M.; Terrassa, J.; Melis, N.; Monerris, M. Parasitism of Bactrocera oleae (Diptera; Tephritidae) by Psyttalia concolor (Hymenoptera; Braconidae) in the Balearic Islands (Spain). J. Appl. Entomol. 2008, 132, 798-805. [CrossRef]

36. Yokoyama, V.Y.; Rendón, P.A.; Sivinski, J. Psyttalia cf. concolor (Hymenoptera: Braconidae) for biological control of olive fruit fly (Diptera: Tephritidae) in California. Environ. Entomol. 2008, 37, 764-773. [CrossRef]

37. Tzanakakis, M.E. Insects and Mites Feeding on Olive: Distribution, Importance Habits Seasonal Development and Dormancy; Brill Academic Publication: Leiden, The Netherlands, 2006; ISBN 978-9004132719.

38. Canale, A.; Benelli, G. Impact of mass-rearing on the host seeking behaviour and parasitism by the fruit fly parasitoid Psyttalia concolor (Szépligeti) (Hymenoptera: Braconidae). J. Pest. Sci. 2012, 85, 65-74. [CrossRef]

39. Fiedler, A.K.; Landis, D.A.; Wratten, S.D. Maximizing ecosystem services from conservation biological control: The role of habitat management. Biol. Control. 2008, 45, 254-271. [CrossRef]

40. Gillespie, M.A.K.; Gurr, G.M.; Wratten, S.D. Beyond nectar provision: The other resource requirements of parasitoid biological control agents. Entomol. Exp. Appl. 2016, 159, 207-221. [CrossRef]

41. Botías, C.; David, A.; Hill, E.M.; Goulson, D. Contamination of wild plants near neonicotinoid seed-treated crops, and implications for non-target insects. Sci. Total. Environ. 2017, 566-567, 269-278. [CrossRef]

42. Cowles, R.S.; Eitzer, B.D. Residues of neonicotinoid insecticides in pollen and nectar from model plants. J. Environ. Hortic. 2017, 35, 24-34. [CrossRef]

43. Benelli, G.; Giunti, G.; Tena, A.; Desneux, N.; Caselli, A.; Canale, A. The impact of adult diet on parasitoid reproductive performance. J. Pest. Sci. 2017, 90, 807-823. [CrossRef]

44. Jervis, M.A.; Kidd, N.A.C. Host feeding strategies in hymenopteran parasitoids. Biol. Rev. 1996, 61, 395-434. [CrossRef]

45. Abram, P.K.; Brodeur, J.; Urbaneja, A.; Tena, A. Nonreproductive effects of insect parasitoids on their hosts. Annu. Rev. Entomol. 2019, 64, 15.1-15.18. [CrossRef]

46. Longley, M.; Jepson, P.C. Effects of honeydew and insecticide residues on the distribution of foraging aphid parasitoids under glasshouse and field conditions. Entomol. Exp. Appl. 1996, 81, 189-198. [CrossRef]

47. Stapel, J.O.; Cortesero, A.M.; Lewis, W.J. Disruptive sublethal effects of insecticides on biological control: Altered foraging ability and life span of a parasitoid after feeding on extrafloral nectar of cotton treated with systemic insecticides. Biol. Control. 2000, 17, 243-249. [CrossRef]

48. Krischik, V.A.; Landmark, A.L.; Heimpel, G.E. Soil-applied imidacloprid is translocated to nectar and kills nectar-feeding Anagyrus pseudococci (Girault) (Hymenoptera: Encyrtidae). Environ. Entomol. 2007, 36, 1238-1245. [CrossRef]

49. Hervé, M.R.; Delourme, R.; Gravot, A.; Marnet, N.; Berardocco, S.; Cortesero, A.M. Manipulating feeding stimulation to protect crops against insect pests? J. Chem. Ecol. 2014, 40, 1220-1231. [CrossRef] [PubMed]

50. Qian, H.; Lu, H.; Ding, H.; Lavoie, M.; Li, Y.; Liu, W.; Fu, Z. Analyzing Arabidopsis thaliana root proteome provides insights into the molecular bases of enantioselective imazethapyr toxicity. Sci. Rep. 2015, 5, 11975. [CrossRef] [PubMed]

51. Serra, A.A.; Couée, I.; Renault, D.; Gouesbet, G.; Sulmon, C. Metabolic profiling of Lolium perenne shows functional integration of metabolic responses to diverse subtoxic conditions of chemical stress. J. Exp. Bot. 2015, 66, 1801-1816. [CrossRef] [PubMed] 
52. Desneux, N.; Rafalimanana, H.; Kaiser, L. Dose-response relationship in lethal and behavioural effects of different insecticides on the parasitic wasp Aphidius. ervi. Chemosphere 2004, 54, 619-627. [CrossRef]

53. Tappert, L.; Pokorny, T.; Hofferberth, J.; Ruther, J. Sublethal doses of imidacloprid disrupt sexual communication and host finding in a parasitoid wasp. Sci. Rep. 2017, 7, 42756. [CrossRef]

54. Hassan, S.A. The initiative of the IOBC/WPRS Working Group on Pesticides and Beneficial Organisms. In Ecotoxicology; Haskell, P.T., McEwen, P., Eds.; Springer: Boston, MA, USA, 1998.

55. Hassan, S.A.; Bigler, F.; Blaisinger, P.; Bogenschütz, H.; Brun, J.; Chiverton, P.; Dickler, E.; Easterbrook, M.A.; Edwards, P.J.; Englert, W.D.; et al. Standard methods to test the side-effects of pesticides on natural enemies of insects and mites developed by the IOBC/WPRS Working Group "Pesticides and Beneficial Organisms". EPPO Bull. 1985, 15, 214-255. [CrossRef]

56. Hassan, S.A. Guidelines for testing the effect of pesticides on beneficial organisms. IOBC/WPRS Bull. 1992, 15, 186.

57. Macfadyen, S.; Banks, J.E.; Stark, J.D.; Davies, A.P. Using semifield studies to examine the effects of pesticides on mobile terrestrial invertebrates. Annu. Rev. Entomol. 2014, 59, 383-404. [CrossRef]

58. Sterk, G.; Hassan, S.A.; Baillod, M.; Bakker, F.; Bigler, F.; Blümel, S.; Bogenschütz, H.; Boller, E.; Bromand, B.; Brun, J.; et al. Results of the seventh joint pesticide testing programme carried out by the IOBC/WPRS Working Group "Pesticides and Beneficial Organisms". BioControl 1999, 44, 99-117. [CrossRef]

59. Talhinhas, P.; Loureiro, A.; Oliveira, H. Olive anthracnose: A yield- and oil quality-degrading disease caused by several species of Colletotrichum that differ in virulence, host preference and geographical distribution. Mol. Plant Pathol. 2018, 19, 1797-1807. [CrossRef] [PubMed]

60. Romero, J.; Agustí-Brisach, C.; Roca, L.F.; Moral, J.; González-Domínguez, E.; Rossi, V.; Trapero, A. A long-term study on the effect of agroclimatic variables on olive scab in Spain. Crop. Prot. 2018, 114, 39-43. [CrossRef]

61. Cacciola, S.O.; Faedda, R.; Sinatra, F.; Agosteo, G.E.; Schena, L.; Frisullo, S.; Magnano di San Lio, G. Olive anthracnose. J. Plant Pathol. 2012, 94, 29-44. [CrossRef]

62. Obanor, F.O.; Jaspers, M.V.; Jones, E.E.; Walter, M. Greenhouse and field evaluation of fungicides for control of olive leaf spot in New Zealand. Crop. Prot. 2008, 27, 1335-1342. [CrossRef]

63. Insecticide Resistance Action Committee (IRAC). Mode of Action Classification. Available online: www.iraconline.org/modes-of-action/ (accessed on 15 September 2020).

64. Jacas, J.A.; Viñuela, E. Side-effects of pesticides on Opius concolor Szèp. (Hymenoptera: Braconidae), a parasitoid of the olive fruit fly. Bull. OILB/IOBC Bull. 1994, 17, 143-146.

65. Ohta, I.; Takeda, M. Acute toxicities of 42 pesticides used for green peppers to an aphid parasitoid, Aphidius gifuensis (Hymenoptera: Braconidae), in adult and mummy stages. Appl. Entomol. Zool. 2015, 50, 207-212. [CrossRef]

66. Harbi, A.; Abbes, K.; Sabater-Muñoz, B.; Beiti, F.; Chermiti, B. Residual toxicity of insecticides used in Tunisian citrus orchards on the imported parasitoid Diachasmimorpha longicaudata (Hymenoptera: Braconidae): Implications for IPM program of Ceratitis capitata (Diptera: Tephritidae). Span. J. Agric. Res. 2017, 15, e1008. [CrossRef]

67. Soderlund, D.M.; Bloomquist, J.R. Neurotoxic actions of pyrethroid insecticides. Annu. Rev. Entomol. 1989, 34, 77-96. [CrossRef]

68. Jacas, J.A.; Viñuela, E. Analysis of a laboratory method to test the effects of pesticides on adult females of Opius concolor (Hym., Braconidae), a parasitoid of the olive fruit fly, Bactrocera oleae (Dip., Tephritidae). Biocontrol. Sci. Technol. 1994, 4, 147-154. [CrossRef]

69. Prabhaker, N.; Morse, J.G.; Castle, S.J.; Naranjo, S.E.; Henneberry, T.J.; Toscano, N.C. Toxicity of seven foliar insecticides to four insect parasitoids attacking citrus and cotton pests. J. Eco. Entomol. 2007, 100, 1053-1061. [CrossRef]

70. Bayram, A.; Salerno, G.; Onofri, A.; Conti, E. Sub-lethal effects of two pyrethroids on biological parameters and behavioural responses to host cues in the egg parasitoid Telenomus busseolae. Biol. Control. 2010, 53, 153-160. [CrossRef]

71. Morais, M.R.; Zanardi, O.Z.; Rugno, G.R.; Yamamoto, P.T. Impact of five insecticides used to control citrus pests on the parasitoid Ageniaspis citricola Longvinovskaya (Hymenoptera: Encyrtidae). Ecotoxicology 2016, 25, 1011-1020. [CrossRef] 
72. Garantonakis, N.; Varikou, K.; Birouraki, A. Comparative selectivity of pesticides used in greenhouses, on the aphid parasitoids Aphidius colemani (Hymenoptera: Braconidae). Biocontrol. Sci. Technol. 2016, 26, 678-690. [CrossRef]

73. Youssef, A.I.; Nasr, F.N.; Stefanos, S.S.; Elkhair, S.S.A.; Shehata, W.A.; Agamy, E.; Herz, A.; Hassan, S.A. The side-effects of plant protection products used in olive cultivation on the hymenopterous egg parasitoid Trichogramma cacoeciae Marchal. J. Appl. Entomol. 2004, 128, 593-599. [CrossRef]

74. Vianna, U.R.; Pratissoli, D.; Zanuncio, J.C.; Lima, E.R.; Brunner, J.; Pereira, F.F.; Serra, J.E. Insecticide toxicity to Trichogramma pretiosum (Hymenoptera: Trichogrammatidae) females and effect on descendant generation. Ecotoxicology 2009, 18, 180-186. [CrossRef]

75. D'Ávila, V.A.; Barbosa, W.F.; Guedes, R.N.C.; Cutler, G.C. Effects of spinosad, imidacloprid, and lambda-cyhalothrin on survival, parasitism, and reproduction of the aphid parasitoid Aphidius colemani. J. Econ. Entomol. 2018, 111, 1096-1103. [CrossRef]

76. Schoonhoven, L.M.; Van Loon, J.J.A.; Dicke, M. Insect-Plant Biology, 2nd ed.; Oxford University Press: Oxford, UK, 2005; p. 421, ISBN 978-0198525943.

77. Desneux, N.; Denoyelle, R.; Kaiser, L. A multi-step bioassay to assess the effect of the deltamethrin on the parasitic wasp Aphidius ervi. Chemosphere 2006, 65, 1697-1706. [CrossRef]

78. Adán, A.; González, T.; Bastante, R.; Budia, F.; Medina, P.; Del Estal, P.; Viñuela, E. Efectos de diversos insecticidas aplicados en condiciones de laboratorio extendido sobre Psyttalia concolor Szèpligeti (Hymenoptera: Braconidae). Bol. San. Veg. Plagas. 2007, 33, 391-397. (In Spanish)

79. Adán, A.; Viñuela, E.; Bengochea, P.; Budia, F.; Del Estal, P.; Aguado, P.; Medina, P. Lethal and sublethal toxicity of fipronil and imidacloprid on Psyttalia concolor (Hymenoptera: Braconidae). J. Econ. Entomol. 2011, 104, 1541-1549. [CrossRef]

80. Kirst, A.H. The spinosyn family of insecticides: Realizing the potential of natural products research. J. Antibiot. 2010, 63, 101-111. [CrossRef]

81. Williams, T.; Javier Valle, J.; Viñuela, E. Is the naturally derived insecticide spinosad ${ }^{\circledR}$ compatible with insect natural enemies? Biocontrol. Sci. Technol. 2003, 13, 459-475. [CrossRef]

82. Biondi, A.; Mommaerts, V.; Smagghe, G.; Viñuela, E.; Zappalà, L.; Desneux, N. The non-target impact of spinosyns on beneficial arthropods. Pest. Manag. Sci. 2012, 68, 1523-1536. [CrossRef] [PubMed]

83. Viñuela, E.; Medina, M.P.; Schneider, M.; González, M.; Budia, F.; Adán, A.; Del Estal, P. Comparison of side-effects of spinosad, tebufenozide and azadirachtin on the predators Chrysoperla carnea and Podisus maculiventris and the parasitoids Opius concolor and Hyposoter didymator under laboratory conditions. Bull. OILB/IOBC Bull. 2001, 24, 25-34.

84. Stark, J.D.; Vargas, R.; Miller, N. Toxicity of spinosad in protein bait to three economically important tephritid fruit fly species (Diptera: Tephritidae) and their parasitoids (Hymenoptera: Braconidae). J. Econ. Entomol. 2004, 97, 911-915. [CrossRef] [PubMed]

85. Biondi, A.; Zappalà, L.; Stark, J.D.; Desneux, N. Do biopesticides affect the demographic traits of a parasitoid wasp and its biocontrol services through sublethal effects? PLoS ONE 2013, 8, e76548. [CrossRef]

86. Grenier, S.; Grenier, A.M. Fenoxycarb, a fairly new insect growth regulator: A review of its effects on insects. Ann. Appl. Biol. 1993, 122, 369-403. [CrossRef]

87. González, M.; Bahena, F.; Viñuela, E. Efectos de distintos Reguladores del Crecimiento de Insectos (RCI) sobre el parasitoide Opius concolor Szèpligeti cuando son ingeridos por la larva huésped. Bol. San. Veg. Plagas. 1998, 24, 193-200. (In Spanish)

88. Hoddle, M.S.; Van Driesche, R.G.; Lyon, S.M.; Sanderson, J.P. Compatibility of insect growth regulators with Eretmocerus eremicus for whitefly control on poinsettias: I. Laboratory Assays. Biol. Control. 2001, 20, 122-131. [CrossRef]

89. Wang, Q.L.; Liu, T.X. Effects of three insect growth regulators on Encarsia formosa (Hymenoptera: Aphelinidae), an endoparasitoid of Bemisia tabaci (Hemiptera: Aleyrodidae). J. Econ. Entomol. 2016, 109, 2290-2297. [CrossRef]

90. Abdallahi, E.; Adán, A.; Viñuela, E. Estudio de la actividad del piriproxifen y fipronil sobre Opius concolor Szèpligeti (Hymenoptera: Braconidae) y su huésped de substitución Ceratitis capitata (Wiedemann) (Diptera: Tephritidae). Bol. San. Veg. Plagas. 2000, 26, 503-512. (In Spanish)

91. Penca, C.; Hodges, A.C. Pyriproxyfen treatment terminates Halyomorpha halys reproductive diapause, with an indirect mortality effect on its egg parasitoid Trissolcus japonicus. J. Pest. Sci. 2017, 90, 1269-1276. [CrossRef] 
92. Madrid, F.J.; Stewart, R.K. Impact of diflubenzuron spray on gypsy moth parasitoids in the field. J. Econ. Entomol. 1981, 74, 1-2. [CrossRef]

93. Glenn, M.; Puterka, G.J. Particle films: A new technology for agriculture. Hort. Rev. 2005, 31, 1-44. [CrossRef]

94. Bengochea, P.; Hernando, S.; Saelices, R.; Adán, A.; Budia, F.; González-Nñez, M.; Viñuela, E.; Medina, P. Side effects of kaolin on natural enemies found on olive crops. Pesticides and Beneficial Organisms. Bull. IOBC/WPRS Bull. 2010, 55, 61-67.

95. Bengochea, P.; Amor, F.; Saelices, R.; Hernando, S.; Budia, F.; Adán, A.; Medina, P. Kaolin and copper-based products applications: Ecotoxicology on four natural enemies. Chemosphere 2013, 91, 1189-1195. [CrossRef]

96. Benhadi-Marín, J.; Pereira, J.A.; Santos, S.A.P. Effects of kaolin particle films on the life span of an orb-weaver spider. Chemosphere 2016, 144, 918-924. [CrossRef]

97. Pascual, S.; Cobos, G.; Seris, E.; González-Núñez, M. Effects of processed kaolin on pests and non-target arthropods in a Spanish olive grove. J. Pest. Sci. 2010, 83, 121-133. [CrossRef]

98. Saour, G.; Makee, H. A kaolin-based particle film for suppression of the olive fruit fly Bactrocera oleae Gmelin (Dip., Tephritidae) in olive groves. J. App. Entomol. 2004, 128, 28-31. [CrossRef]

99. Caleca, V.; Rizzo, R. Tests on the effectiveness of kaolin and copper hydroxide in the control of Bactrocera oleae (Gmelin). IOBC/WPRS Bull. 2007, 30, 111-117.

100. Pascual, S.; Sánchez-Ramos, I.; González-Núñez, M. Repellent/deterrent effect of kaolin and copper on Bactrocera oleae oviposition in the laboratory. IOBC/WPRS Bull. 2010, 59, 83-88.

101. Fungicide Resistance Action Committee (FRAC). Code List: Fungicides Sorted by Mode of Action (Including FRAC Code Numbering). Available online: http://www.phi-base.org/images/fracCodeList.pdf (accessed on 15 September 2020).

102. Jepsen, S.J.; Rosenheim, J.A.; Matthews, C.E. The impact of sulfur on the reproductive success of Anagrus spp. parasitoids in the field. BioControl 2007, 52, 599-612. [CrossRef]

103. Mgocheki, N.; Addison, P. Effect of contact pesticides on vine mealybug parasitoids, Anagyrus sp. near pseudococci (Girault) and Coccidoxenoides perminutus (Timberlake) (Hymenoptera: Encyrtidae). S. Afr. J. Enol. Vitic. 2016, 30, 110-116. [CrossRef]

104. Schaeffer, R.N.; Vannette, R.L.; Brittain, C.; Williams, N.M.; Fukami, T. Non-target effects of fungicides on nectar-inhabiting fungi of almond flowers. Environ. Microbiol. Rep. 2017, 9, 79-84. [CrossRef] [PubMed]

105. Herbicide Resistance Action Committee (HRAC). HRAC Mode of Action Classification 2020. Available online: https://hracglobal.com/files/HRAC_Revised_MOA_Classification_Herbicides_Poster.pdf (accessed on 15 September 2020).

106. Le Bellec, F.; Vélua, A.; Fournier, P.; Le Squinc, S.; Michels, T.; Tendero, A.; Bockstaller, C. Helping farmers to reduce herbicide environmental impacts. Ecol. Indic. 2015, 54, 207-216. [CrossRef]

107. Zimmermann, G. Review on safety of the entomopathogenic fungi Beauveria bassiana and Beauveria brongniartii. Biocontrol. Sci. Technol. 2007, 17, 553-596. [CrossRef]

108. Chandler, D.; Bailey, A.S.; Tatchell, G.M.; Davidson, G.; Greaves, J.; Grant, W.P. The development, regulation and use of biopesticides for Integrated Pest Management. Philos. Trans. R Soc. Lond. B Biol. Sci. 2011, 366, 1987-1998. [CrossRef]

109. Usta, C. Microorganisms in biological pest control-A review (Bacterial toxin application and effect of environmental factors). In Current Progress in Biological Research; Silva-Opps, M., Ed.; IntechOpen: London, UK, 2013; pp. 287-317, ISBN 978-953-51-1097-2. [CrossRef]

110. Alberola, T.M.; Aptosoglou, S.; Arsenakis, M.; Bel, Y.; Delrio, G.; Ellar, D.J.; Ferré, J.; Granero, F.; Guttmann, D.M.; Koliais, S.; et al. Insecticidal activity of strains of Bacillus thuringiensis on larvae and adults of Bactrocera oleae Gmelin (Dipt. Tephritidae). J. Invertebr. Pathol. 1999, 74, 127-136. [CrossRef]

111. Amichot, M.; Curty, C.; Benguettat-Magliano, O.; Gallet, A.; Wajnberg, E. Side effects of Bacillus thuringiensis var. kurstaki on the hymenopterous parasitic wasp Trichogramma chilonis. Environ. Sci. Pollut. Res. 2015, 23, 3097-3103. [CrossRef]

112. Mahmoud, M.F. Pathogenicity of three commercial products of entomopathogenic fungi, Beauveria bassiana, Metarhizium anisopliae and Lecanicillium lecanii against adults of olive fly, Bactrocera oleae (Gmelin) (Diptera: Tephritidae) in the laboratory. Plant Prot. Sci. 2009, 45, 98-102. [CrossRef]

113. Oreste, M.; Baser, N.; Bubici, G.; Tarasco, E. Effect of Beauveria bassiana strains on the Ceratitis capitatePsyttalia concolor system. Bull. Insectol. 2015, 68, 265-272. 
114. Jaronski, S.T.; Lord, J.; Rosinska, J.; Bradley, C.; Hoelmer, K.; Simmons, G.; Osterlind, R.; Brown, C.; Staten, R.; Antilla, L. Effect of Beauveria bassiana-based mycoinsecticide on beneficial insects under field conditions. In The 1998 Brighton Conference-Pests and Diseases; British Crop Protection Council Brighton: Aldershot, UK, 1998; Volume 2, pp. 651-657, ISBN 978-1901396492.

115. Ekesi, S.; Maniania, N.K.; Mohamed, S.A.; Lux, S.A. Effect of soil application of different formulations of Metarhizium anisopliae on African tephritid fruit flies and their associated endoparasitoids. Biol. Control. 2005, 35, 83-91. [CrossRef]

116. Zapata, N.; Medina, P.; González, M.; Budia, F.; Rodríguez, B.; Viñuela, E. Toxicidad de azadirona y 1,7 + 3,7-di-O-acetilhavanensina (4:1) sobre adultos de Psyttalia concolor (Szépligeti) (Hym.: Braconidae). Bol. San. Veg. Plagas. 2004, 30, 783-789. (In Spanish)

117. Rehman, J.U.; Wang, X.; Johnson, M.W.; Daane, K.M.; Jilani, G.; Khan, M.A.; Zalom, F.G. Effects of Peganum harmala (Zygophyllaceae) seed extract on the olive fruit fly (Diptera: Tephritidae) and its larval parasitoid Psyttalia concolor (Hymenoptera: Braconidae). J. Eco. Entomol. 2009, 102, 2233-2240. [CrossRef] [PubMed]

118. Benelli, G.; Canale, A.; Flamini, G.; Cioni, P.L.; Demi, F.; Ceccarini, L.; Macchia, M.; Conti, B. Biotoxicity of Melaleuca alternifolia (Myrtaceae) essential oil against the Mediterranean fruit fly, Ceratitis capitata (Diptera: Tephritidae), and its parasitoid Psyttalia concolor (Hymenoptera: Braconidae). Ind. Crop. Prod. 2013, 50, 596-603. [CrossRef]

119. Alves, T.J.S.; Murcia, A.; Wanumen, A.C.; Wanderley-Teixeira, V.; Teixeira, A.A.C.; Ortiz, A.; Medina, P. Composition and toxicity of a mixture of essential oils against Mediterranean fruit fly, Ceratitis Capitata (Wiedemann) (Diptera: Tephritidae). J. Econ. Entomol. 2019, 112, 164-172. [CrossRef]

120. Kumar, S.M.; Singh, A. Biopesticides: Present status and the future prospects. J. Fertil. Pestic. 2015, 6, 1000e129. [CrossRef]

Publisher's Note: MDPI stays neutral with regard to jurisdictional claims in published maps and institutional affiliations. 